\title{
Self-assembly of ultralarge graphene oxide nanosheets and alginate into layered nanocomposites for robust packaging materials
}

\author{
Revised and re-submitted to: \\ ACS Applied Nano Materials
}

February 2019
SUNNY X. WENG ${ }^{\prime \prime}$, NARIMAN YOUSEFI ${ }^{\prime / *}$, and NATHALIE TUFENKJI
Department of Chemical Engineering, McGill University
3610 University St., Montreal QC
H3A OC5 Canada

"Equal contribution

* Corresponding author: tel: (514) 623-2820; e-mail: nariman.yousefi@mail.mcgill.ca 


\section{Abstract}

The assembly of graphene oxide (GO) nanosheets into bioinspired nacre-like nanocomposites is a potential strategy for the development of robust alternative packaging materials. At sufficiently high concentrations, the self-assembly of ultralarge GO into liquid crystals (LC) in colloidal suspensions with alginate biopolymer is observed. Studying freeze-dried partially-cast nanocomposites reveals the gradual reorientation and merging of these LC domains during evaporation. Evaporation of LC alginate-GO suspensions yields nanocomposite films with highly ordered layered microstructures, and displaying excellent mechanical properties, thermal stability, and moisture barrier performance. Spectroscopic studies and comparison with theoretical models establish the role of hydrogen bonding interactions between the components, and confirm the parallel alignment of ultralarge GO above 2 $\mathrm{wt} \%$, corresponding to the formation of LC domains in aqueous suspension. The insights afforded by this study can help guide the commercial development of strong, environmentally friendly biopolymer-GO nanocomposites. 


\section{Introduction}

Graphene, a two-dimensional arrangement of carbon atoms in a hexagonal lattice, exhibits many remarkable properties; among them, it is one of the strongest materials yet discovered. Though pristine graphene is costly and difficult to produce, graphene oxide $(\mathrm{GO})$ is readily produced via chemical exfoliation of natural graphite. It is possible to restore graphene's electrical conductivity to GO by a subsequent reduction step, forming reduced GO $(\mathrm{rGO}),{ }^{1,2}$ The abundance of hydrophilic functional groups in GO, including hydroxyls and epoxides on the basal plane and carboxyls on the edge, improve its dispersibility in aqueous suspension. ${ }^{1-3}$

GO colloidal suspensions at sufficiently high concentration can form ordered liquid crystals (LC), ${ }^{1,3-5}$ driven by a net increase in entropy as the reduction in the rotational freedom of adjacent nanosheets is exchanged for increased translational freedom in the aligned phase (Figure S1). ${ }^{6-9}$ Since this increase in entropy arises from an excluded-volume effect, the critical concentration for such selfassembly strongly depends on the geometry of the GO. As the lateral size of the nanosheets increases, their movement is further constrained; therefore, the phase transition is observed at much lower concentrations for nanosheets with high aspect ratios. ${ }^{1,7}$ For example, Kim et al. found aqueous suspensions of GO to form nematic LCs above $0.75 \mathrm{wt} \%$ for GO with aspect ratio -700 , and above $0.53 \mathrm{wt} \%$ for GO with aspect ratio $-1600,{ }^{3}$ while Aboutalebi et al. observed an isotropic to nematic phase transition as low as $0.1 \mathrm{wt} \%$ for GO with aspect ratio over $30000 .{ }^{1}$ In fact, with GO of such high aspect ratios, the entropic increase is such that ordering can be achieved even in non-aqueous dispersion..$^{10}$ 
Liquid crystalline GO can be used to fabricate self-aligned GO composite films as the formation of ordered LCs translates into a highly-ordered layered structure upon evaporation or filtration. . $^{1,3,4,8,11}$ This phenomenon has been exploited to produce nanocomposites of GO or rGO and water-based polymers ${ }^{2}$, including epoxies; ${ }^{9}$ polyurethane; ${ }^{8,12}$ chitosan; ${ }^{13,14}$ polydopamine; ${ }^{15-17}$ and alginate. ${ }^{18-23}$ The resulting nanocomposites have a structure that resemble that of nacre, a natural composite comprising calcium carbonate platelets in a biopolymer matrix. This brick-and-mortar structure exhibits extraordinary strength and toughness, arising from the deflection of cracks in the matrix around the platelets, the interface between the two phases, and the ability of the polymer matrix to relieve local stress concentrations around the strong but brittle calcium carbonate. ${ }^{13,24,25}$ Lightweight synthetic nacre-like composites also demonstrate significant robustness and multifunctional properties, ${ }^{14,17,18,20}$ even at relatively low GO content, making them attractive commercial alternatives for high density strong materials such as aluminum and steel.

Furthermore, nacre-inspired layered composites form effective barriers against moisture and gases, as the unique brick-and-mortar nanostructure presents a tortuous path through the polymer, hindering vapour diffusion by blocking a direct path of diffusion through the thickness of the film (Figure S1). ${ }^{12,19,26-30}$ This property of nacre-inspired biocomposites makes them attractive for food packaging and storage, as a more effective and environmentally friendly alternative to currently used multilayer polymer films, which are difficult to recycle. ${ }^{31}$ Such nanocomposites could also prolong the shelf life of packaged food, thereby mitigating economic losses and ecological impacts associated with food waste. 
Alginate is a natural, water-soluble, and biodegradable polymer extracted from seaweed, comprising sequences of $\alpha$-L-guluronic acid and $\beta$-D-mannuronic acid repeating units. Its ease of manufacture and handling, the ample presence of suitable hydrogen bonding sites, and the possibility to cross-link it with multivalent metal ions that can further enhance mechanical performance and barrier properties, render it an attractive polymeric matrix for designing biomimetic GO-reinforced composites. Existing studies of alginate-GO composites focus on compositions with greater than $2 \mathrm{wt} \%$ GO, where dramatic mechanical improvements have been achieved. ${ }^{18,20}$ For example, Ionita et al. achieved a 392\% improvement in Young's modulus and a 59\% improvement in ultimate stress by loading alginate with $6 \mathrm{wt} \%$ GO. However, the mechanical enhancement of composites with GO content lower than $2-3 \mathrm{wt} \%$ is much more modest. ${ }^{18,21}$

We hypothesise that adding alginate to aqueous GO suspensions may increase the critical concentration for transition to a nematic LC phase due to the increased viscosity of the suspension, hydrogen bonding interaction with the GO, and intercalation into the galleries between GO sheets. This would be consistent with observations of GO suspensions with other polymers: Yousefi et al. observed LC formation in waterborne epoxy-GO mixtures containing between 0.5 and 2 wt $\%$ of ultralarge GO (aspect ratio 10 000), with a layered structure clearly visible in cast epoxy-rGO composites at $2 \mathrm{wt} \%$.? They observed a similar threshold of self-alignment in polyurethane-rGO composite films containing $2 \mathrm{wt} \%$ ultralarge rGO nanosheets ${ }^{8}$, with further alignment at $5 \mathrm{wt} \%$ rGO. ${ }^{12}$ Jalili et al. exploited the high aspect ratio of ultralarge GO to form LCs in a variety of organic solvents. This enabled the creation of hybrid structures of ultralarge GO and single-walled carbon nanotubes, which are not dispersible in water. ${ }^{10}$ Although the composition, assembly environment, 
and intended function of the composites produced in their work differ greatly from the present study, a similar strategy of investigation from the perspective of understanding the entire self-assembly process of ultralarge GO in the presence of an aqueous polymer matrix is needed to advance this field. Observing not only the composite properties, but the effect of the aqueous-phase behaviour as a determinant of these properties will provide useful insight into the rational design and optimization of biopolymer-GO composite materials in the future.

In this paper, we investigate the processing and properties of alginate-ultralarge GO nanocomposites, over a broad range of $\mathrm{GO}$ weight contents. After a rapid, one-step evaporation casting process, the LC alginate-GO suspensions self-assemble into layered structures with excellent mechanical and barrier properties. Using a novel method to directly observe the self-assembly of GO through the entire assembly process, we demonstrate that GO content is a key parameter in determining the nanostructure of the composites and therefore their useful properties. Whereas suspensions with low GO content exhibit no LC order and their evaporation-cast films show a mixture of aligned and randomly oriented nanosheets, at high GO content, aqueous LCs form and the evaporation of these suspensions yields densely-layered composite films. The self-assembly of GO nanosheets above a threshold nanosheet content is also reflected in the mechanical properties of the composites, where layered films exhibit significantly larger elastic modulus than their counterparts with less-oriented GO. Finally, we investigate the effect of self-alignment on the barrier properties of the composite films. 


\section{Materials and Methods}

\section{Preparation of ultralarge graphene oxide}

The processing method for GO nanosheets based on Hummers' method ${ }^{32}$ is described elsewhere. ${ }^{9,33,34}$ Briefly, $0.5 \mathrm{~g}$ expanded graphite prepared from graphite flakes (Asbury Carbons) was added to a round-bottom flask in a water bath maintained at $45^{\circ} \mathrm{C} .100 \mathrm{~mL}$ concentrated sulfuric acid and $5.0 \mathrm{~g}$ potassium permanganate (both Sigma Aldrich) were then added to the reaction vessel, and the mixture was stirred for $24 \mathrm{~h}$ under a cold water reflux. $125 \mathrm{~mL}$ of 1:5 hydrogen peroxide (Sigma Aldrich) to deionised (DI) water solution was slowly added to the mixture and stirred for $30 \mathrm{~min}$. Finally, the mixture was washed through successive centrifugation with $1 \mathrm{M}$ hydrochloric acid followed by DI water until the $\mathrm{pH}$ was near neutral. After centrifugation, the aqueous suspensions of GO were diluted to a concentration of $3 \mathrm{mg} / \mathrm{mL}$. A representative SEM micrograph (Figure S2) of the GO is provided in the Supporting Information.

\section{Preparation of composite films}

A $20 \mathrm{mg} / \mathrm{mL}$ alginate solution was prepared by dissolving sodium alginate powder (Sigma Aldrich, A7003) in DI water, and heating to $80^{\circ} \mathrm{C}$ under constant stirring until the sodium alginate powder was fully dissolved. 


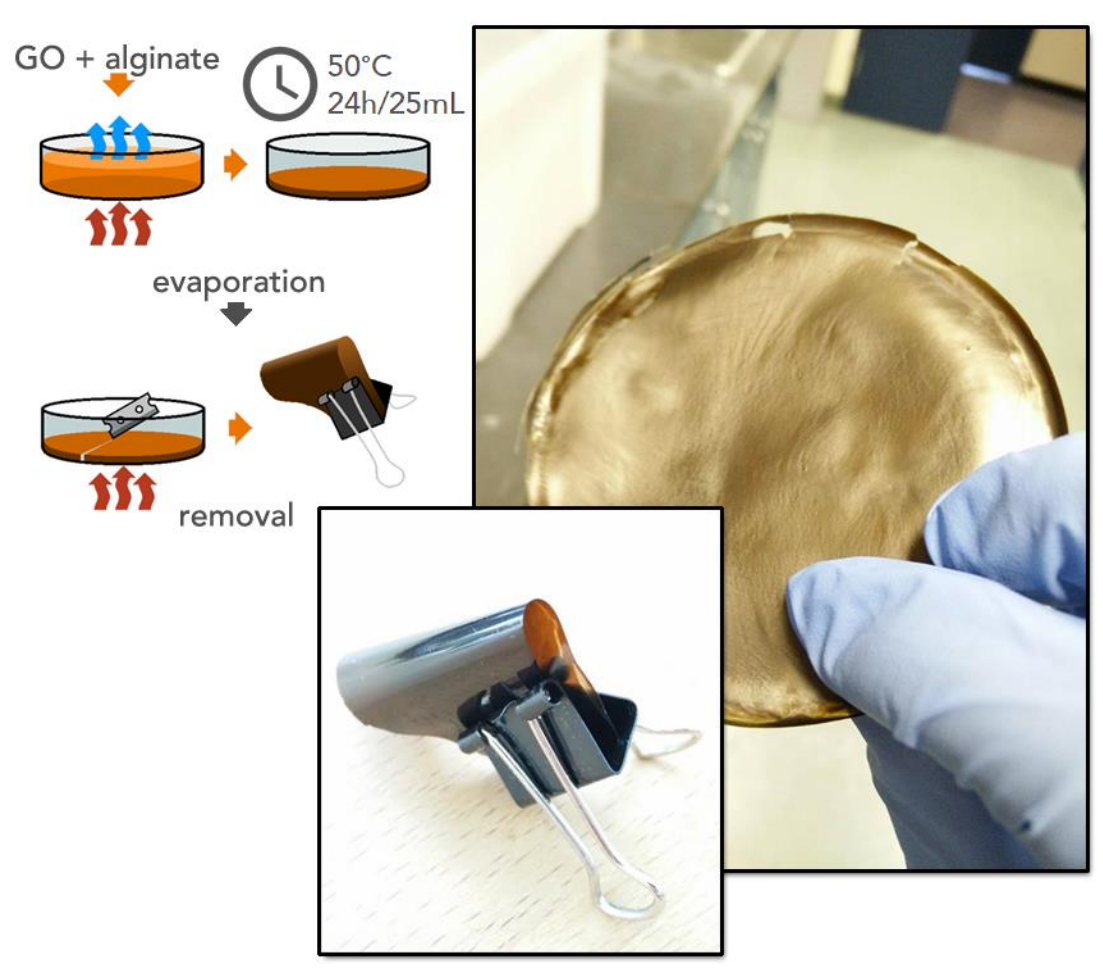

Figure 1 - Schematic representation of the alginate-GO film processing. Using this method, relatively large and flexible films can be prepared.

To produce nanocomposite films with different GO contents, different amounts of the $3 \mathrm{mg} / \mathrm{mL}$ GO suspension were mixed with sodium alginate solution under constant stirring. The mixtures were then placed in a vacuum desiccator for 15 min to remove dissolved air, under constant stirring. Finally, the mixtures were evaporation cast in glass petri dishes of $100 \mathrm{~mm}$ diameter at $50^{\circ} \mathrm{C}$ and $10 \%$ relative humidity until completely dry. Approximately $24 \mathrm{~h}$ of drying was required per $25 \mathrm{~mL}$ of liquid volume. The resulting alginate-GO composite films (Figure 1) were removed by scoring with a razor blade and heating to $60^{\circ} \mathrm{C}$, and stored in closed petri dishes, within the same sealed container and at ambient temperature, until they could be characterised. The compositions of the prepared GO-alginate composites are summarised in Table S1. 
GO control films could not be formed using the evaporation casting method, so they were assembled by vacuum filtration. To produce films of ultralarge GO, $3 \mathrm{mg} / \mathrm{mL}$ GO suspension was filtered through a $0.45 \mu \mathrm{m}$ cellulose acetate membrane (GVS Life Sciences) over a vacuum funnel. The filtered film was then dried in a $50^{\circ} \mathrm{C}$ oven for $24 \mathrm{~h}$ before being separated from the cellulose acetate membrane.

\section{Preparation of aerogels}

Multiple composite suspensions with $6 \mathrm{wt} \% \mathrm{GO}$ were dispensed into $35 \mathrm{~mm}$ glass-bottom petri dishes and cast under the same conditions as the films. These samples were removed at fixed intervals, and stored in a $-80^{\circ} \mathrm{C}$ freezer before being freeze-dried. The freeze-dried aerogels were cut using a razor blade. These samples were observed with a scanning electron microscope (SEM, Hitachi SU3500) to study microstructural evolution over the course of evaporation, as LC GO suspensions self-assembled into layered nanocomposites.

\section{Characterization}

The mechanical properties of the films were studied using dynamic mechanical analysis (DMA, TA Instruments Q800), in film tension mode under ambient conditions. A preload of $0.01 \mathrm{~N}$ was applied to $3 \times 15 \mathrm{~mm}$ rectangular samples, before they were stretched at a constant strain rate of 10 $\mu \mathrm{m} / \mathrm{min}$ until failure. The thickness of each sample was measured using a micrometer, and the strength and modulus calculated using TA Universal Analysis software.

Thermal analysis was performed using simultaneous thermogravimetric analysis (TGA) and differential scanning calorimetry (DSC) (Mettler Toledo TGA/DSC1). The films were cut or 
crumbled into small pieces $\left(-5 \mathrm{mg}\right.$ total sample weight) and heated from 30 to $700^{\circ} \mathrm{C}$ in an alumina pan at a ramp rate of $10^{\circ} \mathrm{C} / \mathrm{min}$, in an inert nitrogen atmosphere. Derivative TGA (DTG) curves were subsequently generated using Mettler Toledo STARe software.

Attenuated total reflectance Fourier-transform infrared (ATR-FTIR, Perkin Elmer Spectrum Two) spectra were collected from $400-4000 \mathrm{~cm}^{-1}$. Raman spectra were collected from $300-3700 \mathrm{~cm}^{-}$ ${ }^{1}$ at a resolution of $0.5 \mathrm{~cm}^{-1}$, using a confocal Raman microscope (Bruker Senterra II) using a $532 \mathrm{~nm}$ laser source at $2 \mathrm{~mW}$ power. Polarised optical micrographs (POM, Nikon SMZ1500 with polariser attachment) were taken by dispensing drops of liquid alginate-GO suspensions onto a glass cover slip. The cross section of films broken under DMA were studied using SEM. Prior to SEM imaging, the films and aerogels were coated with a $9 \mathrm{~nm}$ layer of gold to avoid charge build-up.

Moisture permeability data were collected using a custom apparatus: small squares of selected films $(0.5,2,4,6,10$, and $15 \mathrm{wt} \%$, in triplicate) were affixed with cyanoacrylate glue to a paper gasket with a hole punched out using an ISO 838-standard hole punch (measured diameter $6 \mathrm{~mm}$ ). This assembly was necessary to avoid film warping that was encountered when films were glued directly to the narrow rims of the centrifuge tubes. Each of these assemblies were then glued across the top of 15 $\mathrm{mL}$ centrifuge tubes (Sarstedt) containing $12 \mathrm{~mL}$ DI water, and placed in an oven with temperature and humidity controlled at $37^{\circ} \mathrm{C}$ and $40 \% \mathrm{RH}$, respectively. The mass of each centrifuge tube was measured periodically, and the temporal loss of mass via the evaporation of the water within was recorded. 


\section{Results and Discussion}

\section{LC formation in alginate-GO aqueous suspensions}

Considering the influence of aspect ratio on the formation of GO LCs and the properties of the resulting nanocomposites, ${ }^{35-38}$ we studied the self-assembly behaviour of ultralarge GO with alginate, observing their $\mathrm{LC}$ formation in aqueous suspension and the alignment of the nanosheets into layered structures upon evaporation of the LC suspensions. We also evaluated the mechanical properties and moisture retention of the cast composites. A variety of thermal analysis, spectrometric, and imaging techniques were then used to elucidate the factors influencing the alignment of ultralarge GO in alginate, the understanding of which will help guide the rational design of nacre-like composites for commercial applications.

The rapid, one-step evaporation casting process illustrated in Figure 1 yielded homogeneous composite films that appeared smooth and flexible. In preliminary experiments, we observed that casting smaller volumes of highly-concentrated alginate-GO suspensions resulted in more homogeneous films than casting larger volumes of relatively dilute solutions with the same total polymer and GO content. Similarly, using lower-viscosity sodium alginate (Sigma Aldrich, A1112) also yielded inhomogeneous films, having a mottled 'tortoiseshell' appearance. The difference in quality was particularly pronounced at lower GO content (below $2 \mathrm{wt} \%$; below $4 \mathrm{wt} \%$ using lowviscosity alginate), as the GO tended to separate and agglomerate over the extended casting times necessary for more dilute suspensions. Kim et al. observed similar phase separation in lowconcentration aqueous $\mathrm{GO}$ suspensions below $0.6 \mathrm{wt} \%$, and reported the formation of a dense nematic 
phase below an isotropic phase. ${ }^{3}$ In contrast, our viscous concentrated alginate-GO suspensions were metastable, remaining uniform over several days, well in excess of the casting time.
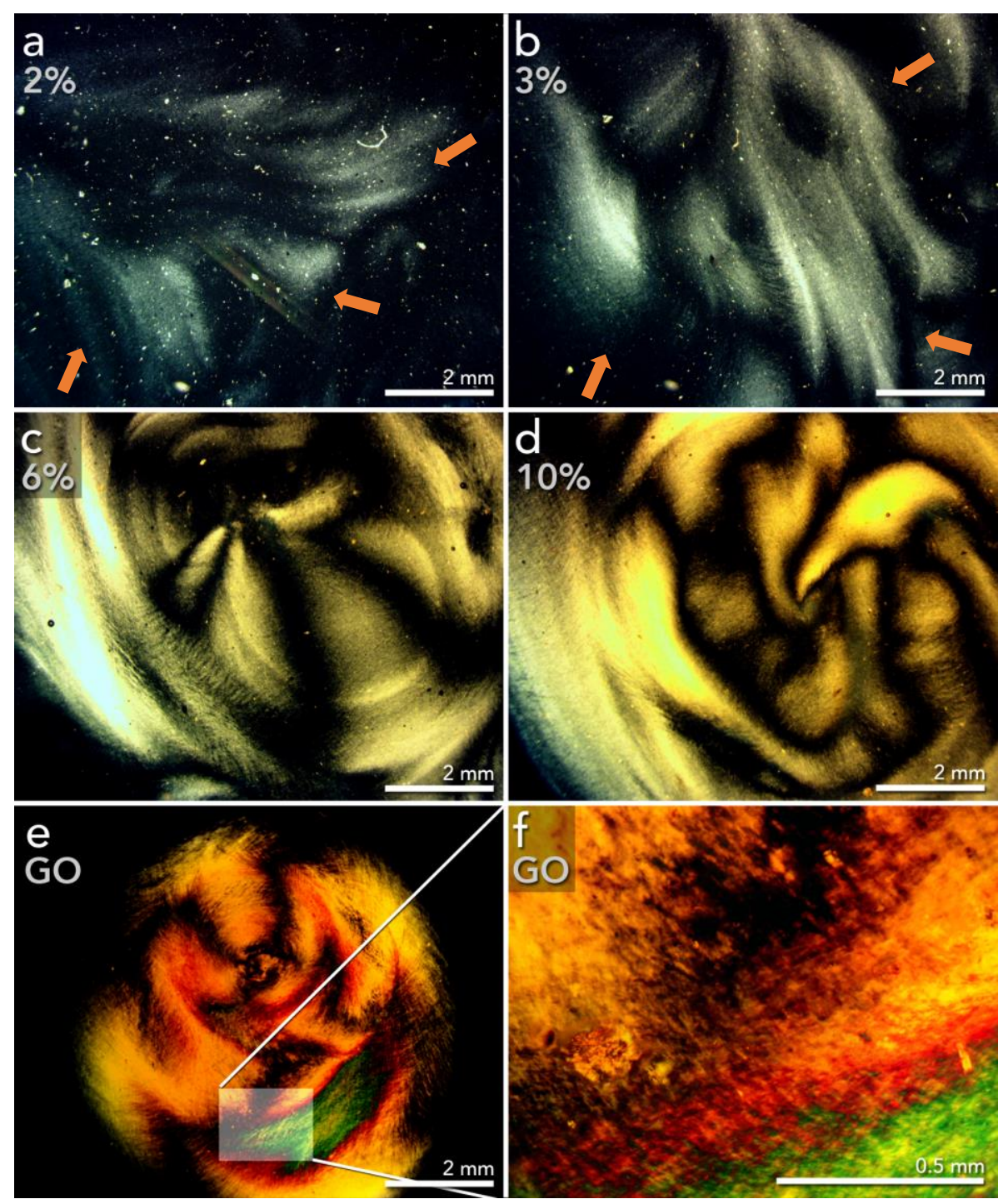

Figure 2 - Polarised optical micrographs of alginate-GO suspensions. As $G O$ content increases, birefringent liquid crystalline domains (light coloured) form. (a) 2 wt\% GO, (b) 3 wt\% GO, (c) 6 wt\% GO, (d) 10 wt\% GO, (e) $3 \mathrm{mg} / \mathrm{mL} \mathrm{GO},(f)$ higher magnification image of $G O$.

To illustrate the self-assembly behaviour of ultralarge GO at different concentrations within alginate, polarised optical micrographs were taken (Figure 2) of selected alginate-GO liquid suspensions $(2,3,6,10 \mathrm{wt} \%$ in suspension, and $3 \mathrm{mg} / \mathrm{mL} \mathrm{GO})$. Under crossed polarisers, the 
isotropic-nematic phase transition manifests as birefringent regions at $3 \mathrm{wt} \%$, which expand through the entire sample when the GO content is increased to $6 \%$. This concentration is notably higher than that observed for GO LC formation in water ${ }^{1}$, and for ultralarge GO-epoxy dispersions. ${ }^{9}$ The formation and expansion of these birefringent regions confirm the self-assembly of GO sheets into a lyotropic nematic phase.

\section{Structural characterization}

To examine the effect of LC formation on the microstructure of the cast films, SEM images were taken of the fracture cross-sections of alginate-GO nanocomposites (Figure 3). The micrographs demonstrate that upon evaporation, the ultralarge GO sheets indeed form layered structures within the alginate matrix. At very low GO content (0.5 and $1 \mathrm{wt} \%$ ), domains of less-aligned (Figure S3b) and highly-aligned (Figure 3b) GO are interspersed throughout the polymer matrix. As the GO content increases to $2 \%$, the highly-aligned layered structure is present throughout the entire sample, and the layers become markedly more tightly-packed and well-ordered as the GO content further increases (Figure 3c-e). At 6\%, a very dense and uniform layered structure is observed through the entire cross section, which is clearly visible in the low-magnification SEM (Figure 3a). The concentration range over which this uniform aligned structure of densely-packed nanosheets appears corresponds to that at which LC domains emerged in the alginate-GO liquid suspensions (Figure 2). 


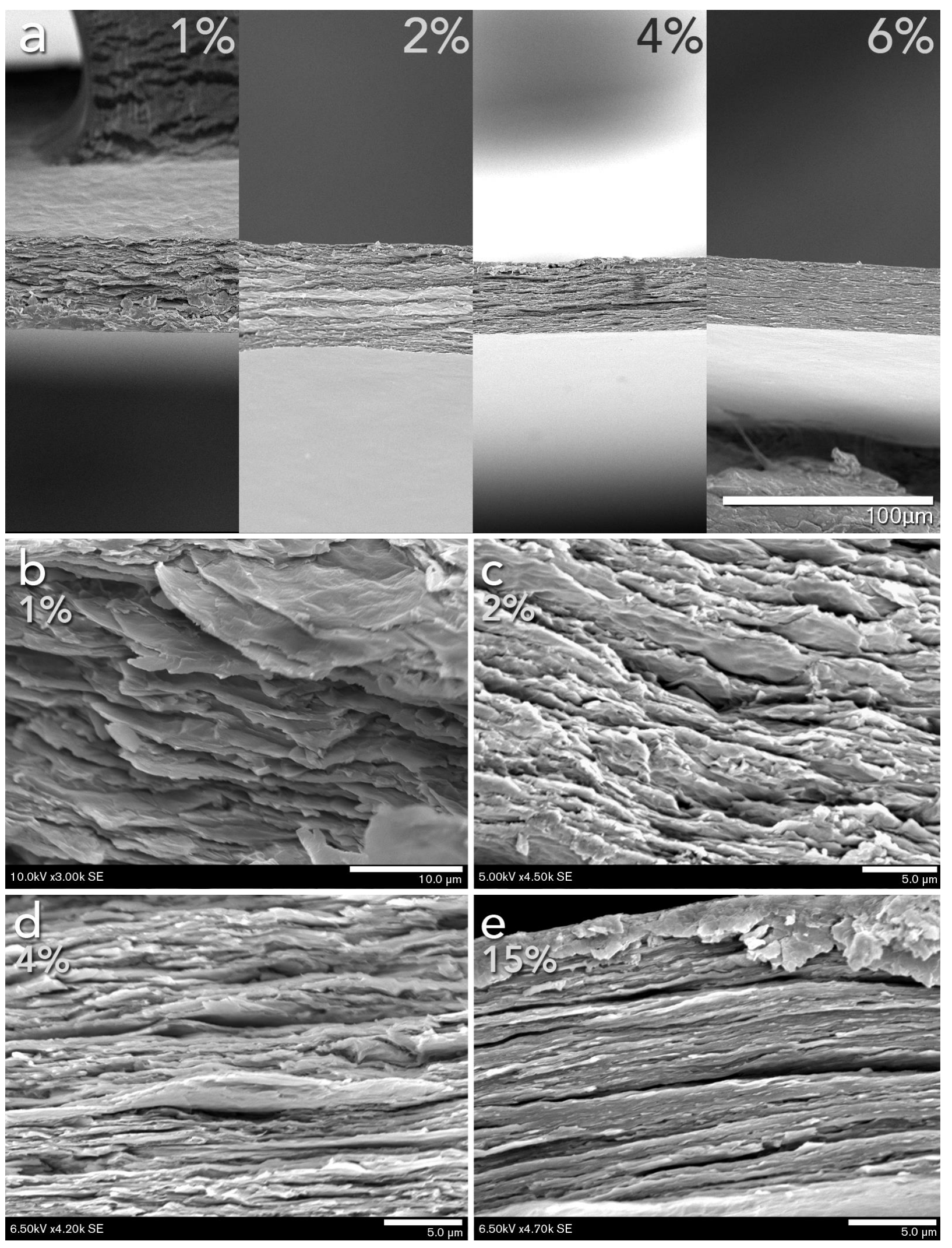

Figure 3-Low- (a) and high- (b-e) magnification SEM images of fracture cross-sections. With increasing GO content, a gradual densification and alignment of $G O$ sheets into a well-ordered layered structure is observed. 
To better understand the transformation of alginate-GO LC domains in the aqueous suspension into layered nacre-like nanocomposite films, the microstructures of the mixtures were studied at various intervals over the course of the evaporative casting process. Observing the aerogels produced by freeze-drying partially-cast alginate-GO suspensions provides further insight into the behaviour of the GO sheets through the casting process. Because of the random 3D orientation of $\mathrm{LC}$ domains in suspension, both the top surface and the cross sections of the samples were studied by SEM (Figure 4). Since the preparation of the cross-sectional samples by cutting with a razor blade may have deformed the microstructure, the top-down images provide a clearer indication of the LC structure of the samples taken at the early stages of film formation $(0.5-3 \mathrm{~h})$.

Figure 4a provides a low-magnification top-down view of the aerogels. The formation of polymercoated GO sheets into many differently-oriented LC domains is strikingly evident in the $1 \mathrm{~h}$ and $3 \mathrm{~h}$ samples, similar to those observed in the low-GO cast films. The high-magnification top-down images in Figure $4 \mathrm{~b}$ show that as evaporation proceeds, the spacing between the layers in these domains decreases, and the wisps of alginate (marked with arrows) bridging the sheets become thicker and denser.

At longer time scales, the LC domains reorient from a random 3D orientation into uniform layers, and a skin forms on the surface of the samples (Figure 4a, $15 \mathrm{~h}$ ), making top-down observation difficult. In cross section (Figure 4c), a similar trend is observed: from $0.5-3 \mathrm{~h}$, the density of the polymercoated GO greatly increases; by $15 \mathrm{~h}$, a continuous alginate matrix has formed, with the aligned GO sheets (Figure 4c, $15 \mathrm{~h}$ ) visible when they protrude. From these images, the process by which the films' layered structure develops becomes clear. At sufficient concentration, LC domains form in the 
alginate-GO system. As the evaporating water reduces the spacing between layers within these ordered regions, the discrete domains themselves grow and merge. As a large, ordered structure emerges throughout the material, the layers finally become oriented in the same plane, normal to the direction of evaporation, resulting in the uniform self-assembled microstructure observed in the films. Highmagnification top-down observations of cracks in a sample cast for $15 \mathrm{~h}$ (Figure $4 \mathrm{~d}$ ) and of a fullyformed film (Figure 4e) illustrate the merging of alginate and GO sheets at $15 \mathrm{~h}$, filling pores vacated through evaporation, to form a uniform, solid and non-porous film at $25 \mathrm{~h}$. 

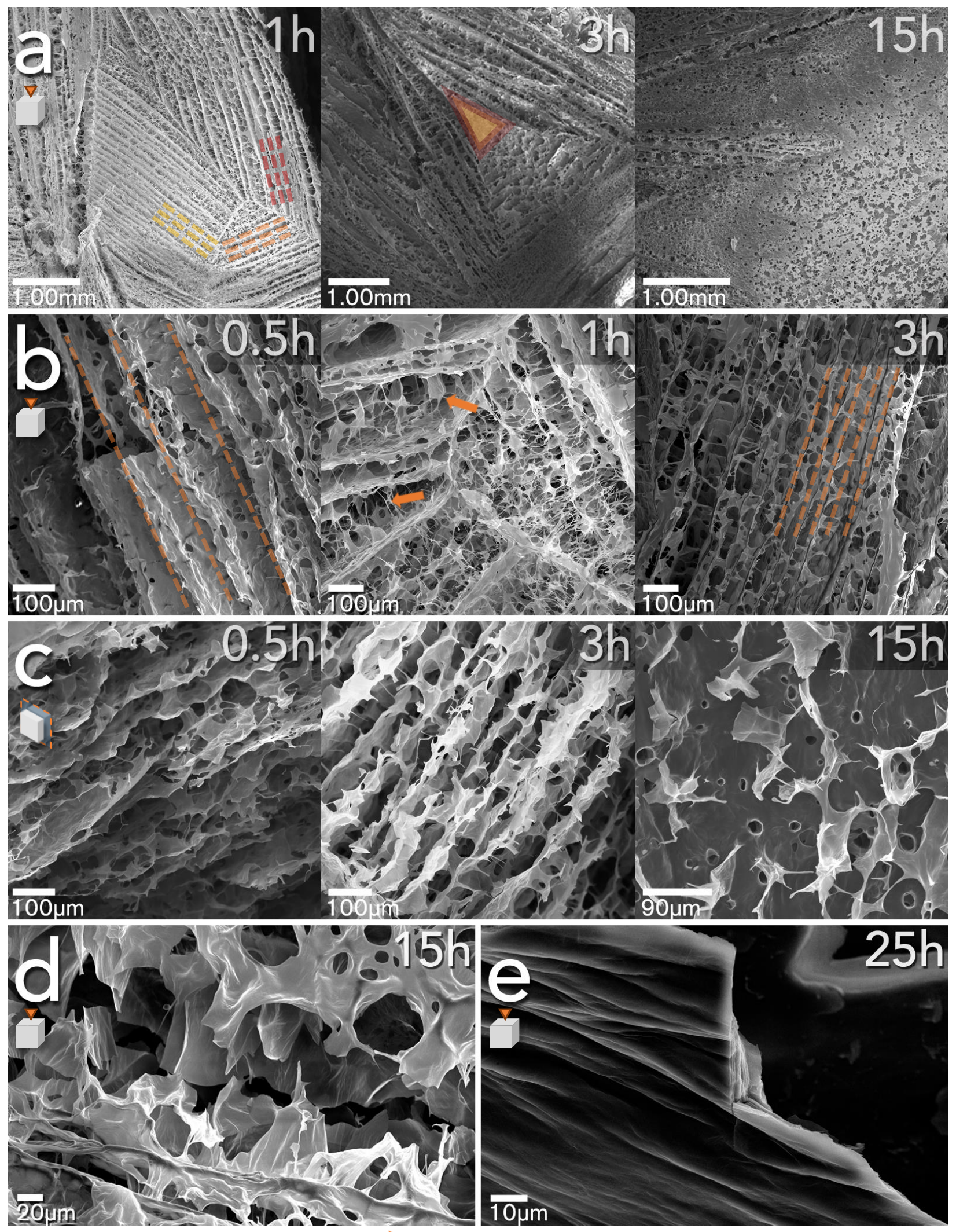

Figure 4-Top down $\nabla$ and cross-sectional SEM images of freeze-dried alginate-GO aerogels. Low- (a) and high-magnification $(b, c)$ views show the merging of $L C$ domains, and the densification of layers within those domains, as evaporation proceeds. Detail $(\boldsymbol{d}, \boldsymbol{e})$ views show that at long time scales, layers are oriented in the same plane, normal to the direction of evaporation. 


\section{Mechanical properties}

Self-alignment of a strong anisotropic nanomaterial such as GO can result in superior mechanical properties along the direction of alignment in the resulting alginate-GO nanocomposites. Indeed, the concentration range over which LC formation was observed in suspension, and over which self-aligned layered structures were observed in the cast films, is consistent with a previous study on mechanical enhancements in evaporation-cast alginate-GO composites..$^{18}$ Ionita et al. did not examine composites with greater than $6 \mathrm{wt} \% \mathrm{GO}$, and liquid crystallinity was not mentioned as a possible mechanism of self-assembly. On the other hand, Chen et al. explored vacuum-filtered, rather than evaporation-cast, alginate-GO films from 13.5 to 77.4 vol\% GO (corresponding to $-25-85$ wt $\%$ GO) where they observed increases in ultimate stress and elastic modulus up to $20-25 \mathrm{vol} \%(-35-40 \mathrm{wt} \%)$, which is a substantially higher GO content than the phase transition concentration threshold observed in our work (Figures 2 and 3). The difference in assembly method could in part explain the discrepancy: the self-assembly in the former study ${ }^{18}$ is driven by evaporation and gravity, whereas the addition of vacuum pressure in the latter study ${ }^{20}$ may have disrupted the self-assembly process of LC phases. Moreover, the authors ${ }^{18,20}$ do not report the dimensions of GO nanosheets; as the formation of discotic nematic LCs depends strongly on the geometrical anisotropy of the constituent platelets ${ }^{1,3-9}$, this factor will also significantly impact the self-assembly into mechanically-strong layered nanostructures. 

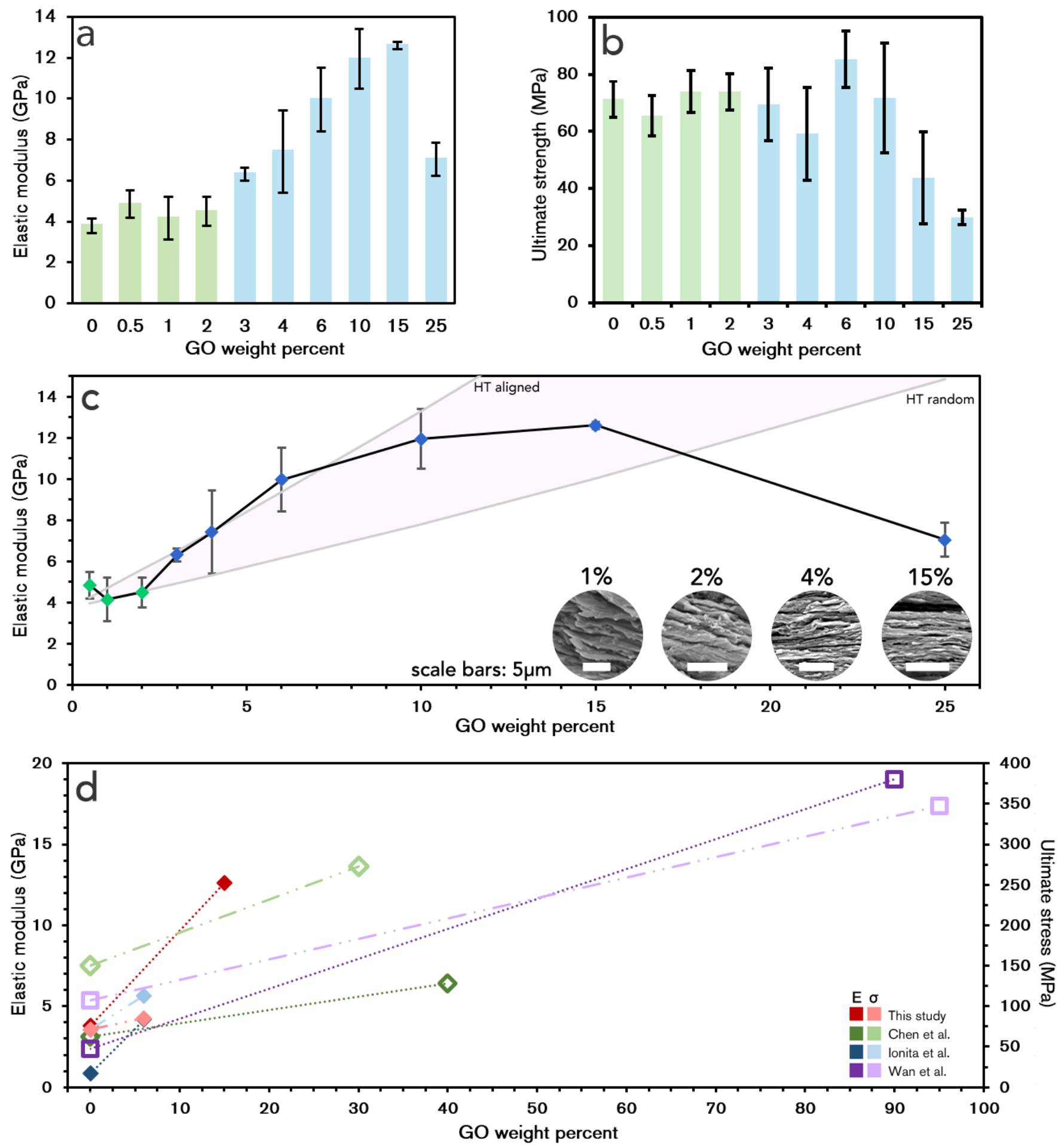

Figure 5-Mechanical properties of alginate-GO nanocomposite films. Evolution of elastic modulus (a) and tensile strength (b) with GO content. (c) Comparison of observed moduli against those predicted by the Halpin-

Tsai model. (Inset: SEM of selected cross-sections illustrates evolution of GO alignment.) (d) Comparison of elastic modulus ( $E$ ) and ultimate stress $(\sigma)$ with those reported in literature. (Improvements in modulus and ultimate stress are shown with dotted and dashed lines, respectively. Symbols $\$$ : alginate; $\mathbf{m}$ : chitosan; open: vacuum filtered; filled: evaporation cast) 
Tensile tests of our alginate-GO nanocomposite films demonstrate that the elastic modulus (Figure 5a) in the low GO content films (up to $2 \mathrm{wt} \% \mathrm{GO}$ ) does not significantly increase in comparison with that of the neat polymer $(3.79 \mathrm{GPa}$, Table $\mathrm{S} 1)$. However, from $3 \mathrm{wt} \% \mathrm{GO}$ upwards, much greater reinforcement is demonstrated as the GO sheets self-assemble into a uniform layered structure as observed by SEM. The onset of stiffening again corresponds to the concentrations where the nematic phase emerged in suspensions of ultralarge GO with alginate. As the GO content reaches $15 \mathrm{wt} \%$, an elastic modulus of $12.59 \pm 0.19 \mathrm{GPa}$ is achieved, representing a $230 \%$ improvement over the neat alginate. Above this GO concentration, the films become brittle, failing at very low strain levels, and the elastic modulus of the $25 \mathrm{wt} \%$ film is significantly decreased. Working with vacuumassembled alginate-GO composites, Chen et al. reported a similar trend of improved elastic modulus with increased GO content, with a maximum of $6.5 \mathrm{GPa}$ at 25.5 vol\% $(-40 \mathrm{wt} \%)$ before the mechanical properties decreased again at very high GO content. ${ }^{20}$ Casting alginate-GO films with an additional thermal post-treatment, Ionita et al. also achieved similar enhancements in mechanical properties, measuring a maximum respective ultimate strength and elastic modulus of $4.18 \mathrm{GPa}$ and $113 \mathrm{MPa}$ (from $0.85 \mathrm{GPa}$ and $71 \mathrm{MPa}$ ) at $6 \mathrm{wt} \% \mathrm{GO}$, which was the highest GO content evaluated. ${ }^{18}$

The maximum measured elastic modulus in this study is among the highest reported for biopolymer-GO composites at any GO content (Figure 5d), with only one report of a higher modulus in a vacuum-assembled chitosan composite with a much higher GO content of $90 \mathrm{wt} \% .{ }^{14}$ Compared to existing alginate-GO composites, the stiffness of our nanocomposites exceeds those previously achieved in either vacuum-assembled or evaporation-cast films, while maintaining a relatively low GO content, as a result of the ultralarge size of our GO nanosheets. 
The Halpin-Tsai micromechanical model predicts the elastic modulus of fibre-reinforced composites. ${ }^{35,36}$ As demonstrated by van Es, the Halpin-Tsai model can be modified to predict the stiffness of platelet-reinforced composites ${ }^{36,37}$; the use of this corrected model to study GO-reinforced composites is well-established. ${ }^{12,20,38}$ As the model can predict the stiffness of an ideal nanocomposite with either a randomly dispersed or an aligned anisotropic nanofiller, it is also useful to quantify the degree of alignment of ultralarge GO in the as-cast composite films by comparing the measured and predicted moduli.

The Halpin-Tsai model yields the modulus $E_{C}$ of a composite with randomly-oriented filler and the modulus $E_{P}$ of a composite with perfectly aligned filler, respectively, as:

$$
\begin{gathered}
\frac{E_{C}}{E_{M}}=\frac{3}{8} \cdot \frac{1+\xi \eta_{L} V_{F}}{1-\eta_{L} V_{F}}+\frac{5}{8} \cdot \frac{1+2 \eta_{T} V_{F}}{1-\eta_{T} V_{F}} \\
\frac{E_{P}}{E_{M}}=\frac{1+\xi \eta_{L} V_{F}}{1-\eta_{L} V_{F}} \\
\eta_{L}=\frac{\left(E_{F} / E_{M}\right)-1}{\left(E_{F} / E_{M}\right)+\xi} \\
\eta_{T}=\frac{\left(E_{F} / E_{M}\right)-1}{\left(E_{F} / E_{M}\right)+2}
\end{gathered}
$$

where $E_{F}$ and $E_{M}$ are the respective Young's moduli of the filler and matrix; $V_{F}$ is the volume fraction of $\mathrm{GO}$ in the composite; and $\xi$ is a shape factor related to the aspect ratio of the filler.

The respective densities of alginate and GO are 1.38 (experimentally determined by casting alginate solution and measuring the weight and volume of the solid mass) and $2.20 \mathrm{~g} / \mathrm{cm}^{3} .38$ The elastic modulus of alginate was measured to be $3.79 \mathrm{GPa}$, and the stiffness of GO is reported to be 145.3 N/m. ${ }^{39}$ Finally, van Es's corrected shape factor $\xi=2 \alpha / 3$ - where $\alpha$ is the aspect ratio of our 
ultralarge GO (10 000) — was used to calculate the predicted elastic moduli of aligned and randomly dispersed alginate-GO nanocomposites in this work (Figure 5c).

These calculated moduli show strikingly good agreement when compared against the experimentally observed values. The gradual alignment of the ultralarge GO into a well-aligned structure is observed from 2 to $3 \mathrm{wt} \% \mathrm{GO}$, as the observed moduli transition between the predictions for randomly-oriented and unidirectional fillers, corroborating both the SEM microstructural observations and the observation of LC formation under POM. Above $6 \mathrm{wt} \% \mathrm{GO}$, the observed elastic modulus increases at a lower rate, falling below that predicted by the model for a biphasic aligned structure; this deviation from the predicted modulus coincides with a measured decrease in toughness observed between 6 and 10 wt\% (Table S1). The Halpin-Tsai model assumes an ideal two-phase composite, with each filler particle perfectly enveloped by the matrix ${ }^{35}$ leading to perfect bonding between the two phases. Where the filler-matrix interface has significantly different properties than either phase, this assumption fails, with the interface effectively acting as a weakened third phase. This could occur in the case of weak interfacial bonding that cannot transfer tensile loads that the nanofiller itself would otherwise support. The presence of defects in the microstructure, such as the inclusion of moisture or air, would also cause deviation from the ideal two-phase structure. 


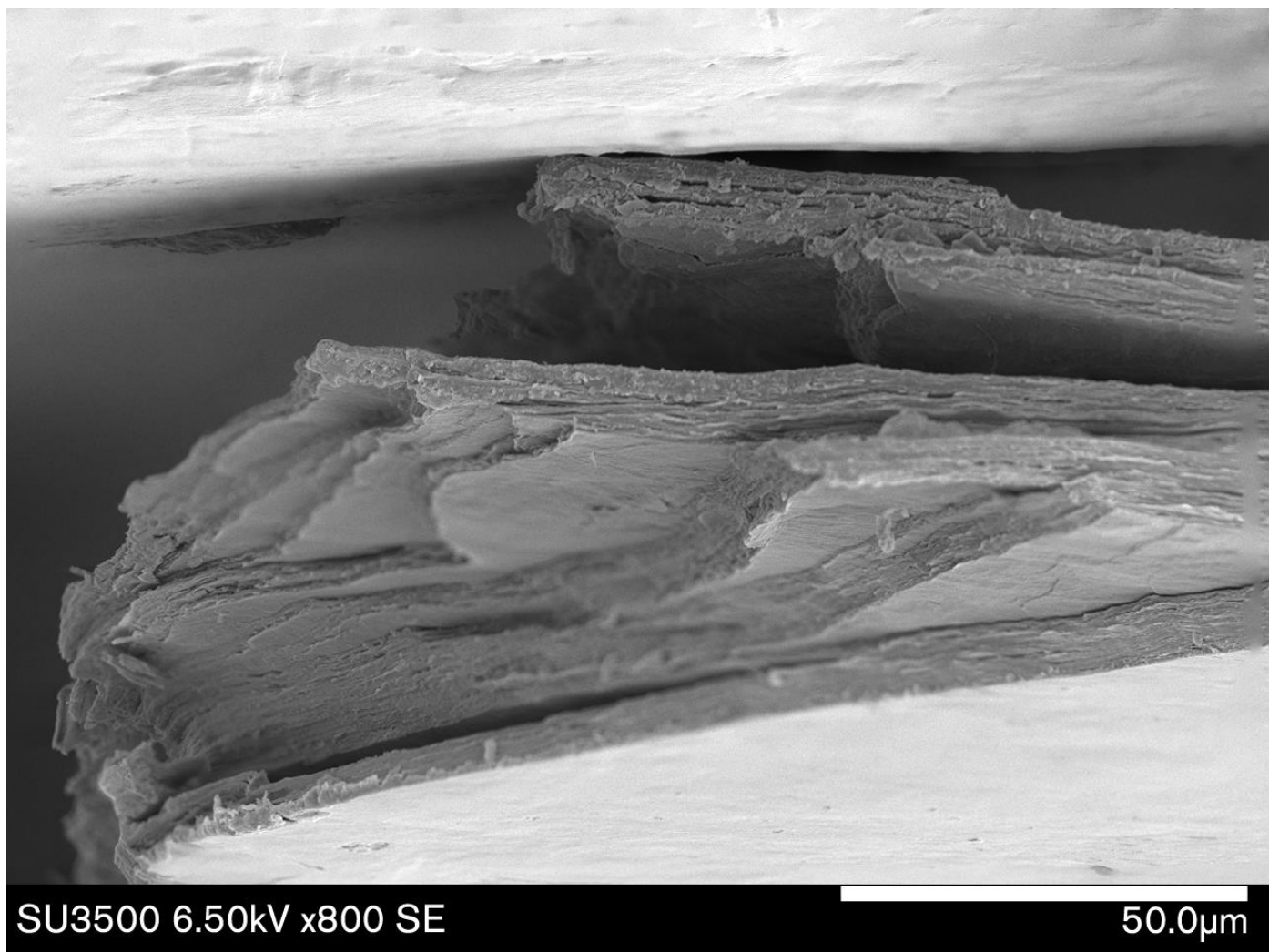

Figure 6 - Low-magnification SEM of fracture (15 wt\% GO) taken at oblique angle. Multiple exposures stacked to provide deep depth of field (see Supporting Information).

The observed improvements in elastic modulus are achieved with minimal decrease in the tensile strength of the nanocomposites. The ultimate tensile strength (at fracture) of the nanocomposites does not change significantly below $15 \mathrm{wt} \% \mathrm{GO}$, with modest improvements achieved up to 6\% (Figure 5b). However, at very high GO content, the films become brittle and eventually the tensile strength decreases to less than that of the neat polymer. In general, we observed that the toughness of the composites decreases with increasing GO content, with the $0.5 \%$ film being the only exception (Table S1). As synergistic mechanical enhancement can only be effective when there is an efficient stress transfer between the GO and alginate, the strength of the interfacial bonding is crucial in determining the performance of the nanocomposite. Structural defects created during the evaporation process, such as GO aggregation or entrapment of water in the highly aligned structure, could thus have contributed to the weakening of the films at very high GO contents. 
A close inspection of the freeze-fractured cross-section of the $15 \%$ film (Figure 6) reveals that cracks propagate through interlayer delamination, and traces of crack deflection are minimal. The dominant delamination fracture behaviour, indicative of relatively weak interfacial bonding, explains the decreasing toughness of the nanocomposite films. Considering the thermal and spectroscopic analyses described below, we conjecture that this reduced interfacial strength is due to intercalation of moisture into the layered structure formed by the GO, interfering with the alginate-GO bonding.

Compton et al. investigated the effect of moisture content in $\mathrm{GO}$ and polymer-GO films assembled by vacuum filtration. ${ }^{40}$ The intercalation of water molecules into pristine GO paper was found to enhance the mechanical performance through the creation of hydrogen bonding networks. The stiffness of the GO paper increased with water content until the available hydroxyl and epoxy groups were saturated; additional water above this level severely weakened the materials. However, in PVA-GO composites, the stiffness continuously improved as water content was reduced, up to the point where no further dehydration was experimentally achievable. The authors demonstrate that the intercalated polymer chains in the nanocomposite film participate in hydrogen bonding with the GO sheets, replacing the role of water in the pristine GO papers. However, they suggest that very small amounts of moisture, below experimentally achievable levels, could still enhance the mechanical performance of polymer-GO nanocomposites if water molecules are able to bond with GO functional groups that are inaccessible to bulkier polymer chains.

\section{Spectroscopic analysis}

Spectroscopic analysis can provide more insight into the nature of the physical and/or chemical interactions that occur between the abundant functional groups on both GO and alginate. The Raman 
spectrum of ultralarge GO presented in Figure $S 4$ shows the $D$ and $G$ bands at $\sim 1350 \mathrm{~cm}^{-1}$ and $\sim 1600$ $\mathrm{cm}^{-1}$ respectively, with the relative peak intensity ratio $I_{D} / I_{G}$ of approximately 1.0 . The location of the peaks is consistent with previous observations of $\mathrm{GO}^{14,17,20,41-44}$, with their relative intensity relatively lower. ${ }^{14,17,20}$ Since the $G$ band is associated with sp $^{2}$ carbon in the basal plane of GO and the $D$ band is an indication of $\mathrm{sp}^{3}$ carbon which arises from edges and defects in the carbon lattice ${ }^{41,43,45,46}$, the low intensity ratio can be interpreted as an indication of the presence of few defects in, the ultralarge GO sheets.

The Raman spectra of the alginate-GO composite films show a similar $I_{D} / I_{G}$ to the GO itself, with a mean near 0.90 ; between the different composite formulations, there is no significant difference in the positions of the $D$ and $G$ peaks or in their relative intensity (Figure S4, inset). This suggests that alginate does not significantly alter the structure of ultralarge GO, with primarily non-covalent interactions such as hydrogen bonding taking place. In contrast, changes in the $I_{D} / I_{G}$ ratio are expected when GO sheets participate in stronger covalent bonding with the polymer matrix due to the formation of $\mathrm{sp}^{3}$ domains. ${ }^{47}$ 


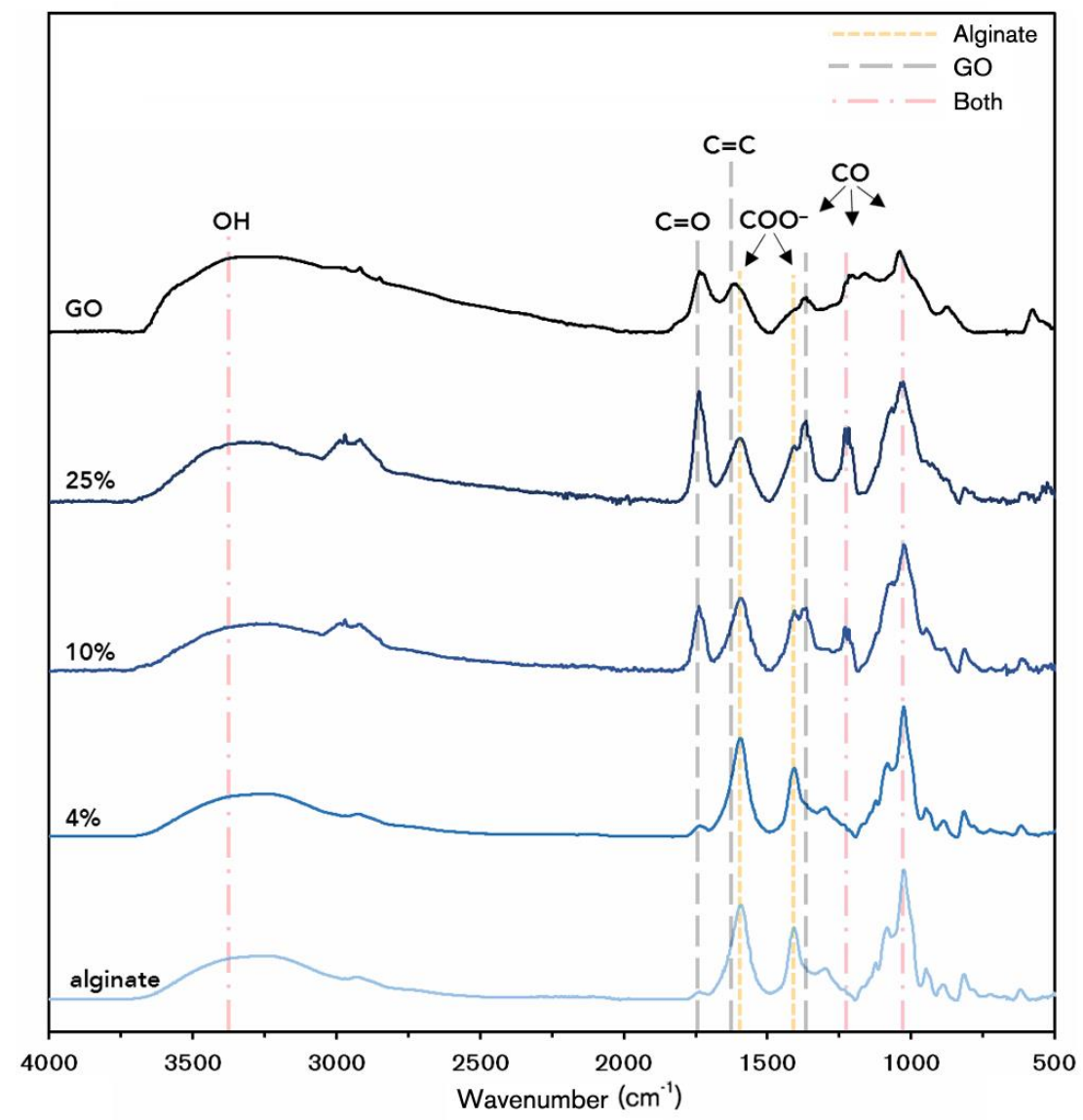

Figure 7-Representative FTIR spectra of alginate-GO nanocomposite films.

The FTIR spectra in Figure 7 further indicate the nature of the interfacial interactions between the GO nanosheets and the alginate. The peak assignments are summarised in Table S2.44,48-52 The $\mathrm{C}=\mathrm{O}$ stretching band at $1740 \mathrm{~cm}^{-1}$, and the carboxyl- and epoxide-associated $\mathrm{CO}$ stretching bands at 1360 and $1240 \mathrm{~cm}^{-1}$ respectively, each show a marked increase in intensity in the composite films. A characteristic red-shift is visible in the bands attributed to the alginate-associated carboxyl groups at 1600 and $1410 \mathrm{~cm}^{-1}$, with the broad $\mathrm{OH}$ stretching band also shifting to lower frequencies. This peak also intensifies and widens with increasing GO content, which is attributed to the increased moisture content in these composites. ${ }^{51}$ Overlapping with this water-associated peak, another signal emerges near $2900 \mathrm{~cm}^{-1}$ which is associated with the stretching vibration of $\mathrm{CH}$ bonds in the graphene sheets. ${ }^{51,52}$ Hence, this signal also strengthens with GO content, before being overwhelmed by the 
stronger water-associated peak in the GO control film. Taken together, the spectra support the presence of trapped water in the high-GO films, and confirm the occurrence of the anticipated hydrogen bonding between the two components at oxygen-bearing functional groups. ${ }^{53,54}$

\section{Thermal analysis}

Thermal analysis of the alginate-GO nanocomposites demonstrates that films with increased GO content generally exhibit improved thermal stability over the alginate control (Figure 8a), with the overall rate of degradation decreasing in the region above $200^{\circ} \mathrm{C}$ where alginate decomposes. ${ }^{55}$ The addition of up to $6 \mathrm{wt} \%$ of GO slightly increases the $25 \%$ decomposition temperature (Table 1 ). Conversely, from $10 \mathrm{wt} \%$ upwards, the initial degradation becomes more rapid and the $25 \%$ decomposition temperature is reduced. The opposite effect is observed for the $50 \%$ decomposition temperature, which decreases in the 6 and $10 \mathrm{wt} \%$ films, but drastically increases at higher GO loading to $20-25^{\circ} \mathrm{C}$ above that of the neat polymer.

\begin{tabular}{ccc}
\multicolumn{3}{c}{ Table $\mathbf{1}-$ Thermal analysis data } \\
\hline Material & $\mathbf{2 5 \%}$ deg. $\left({ }^{\circ} \mathrm{C}\right)$ & $\mathbf{5 0 \%}$ deg. $\left({ }^{\circ} \mathrm{C}\right)$ \\
\hline Alginate & 228.5 & 343.0 \\
$2 \%$ & 232.3 & 343.5 \\
$6 \%$ & 230.0 & 328.6 \\
$10 \%$ & 223.5 & 329.1 \\
$15 \%$ & 220.4 & 369.9 \\
$25 \%$ & 189.9 & 363.7 \\
GO-V & 199.9 & - \\
\hline
\end{tabular}

These results can be explained by two phenomena, which are more clearly illustrated by the DTG curves (Figure 8b). Here, high GO content films show a rightward broadening of the lower primary degradation peak, initially centred near $240^{\circ} \mathrm{C}$. As more $\mathrm{GO}$ sheets are introduced into the nanocomposites, polymer chains intercalate into the densely packed layers and become constrained, ${ }^{37,38}$ causing an increase in the decomposition onset temperature. Further, the self-aligned 
ultralarge GO sheets themselves form an effective barrier against the diffusion of gases produced as the polymer degrades, contributing to the rightward shift of the primary degradation peak.
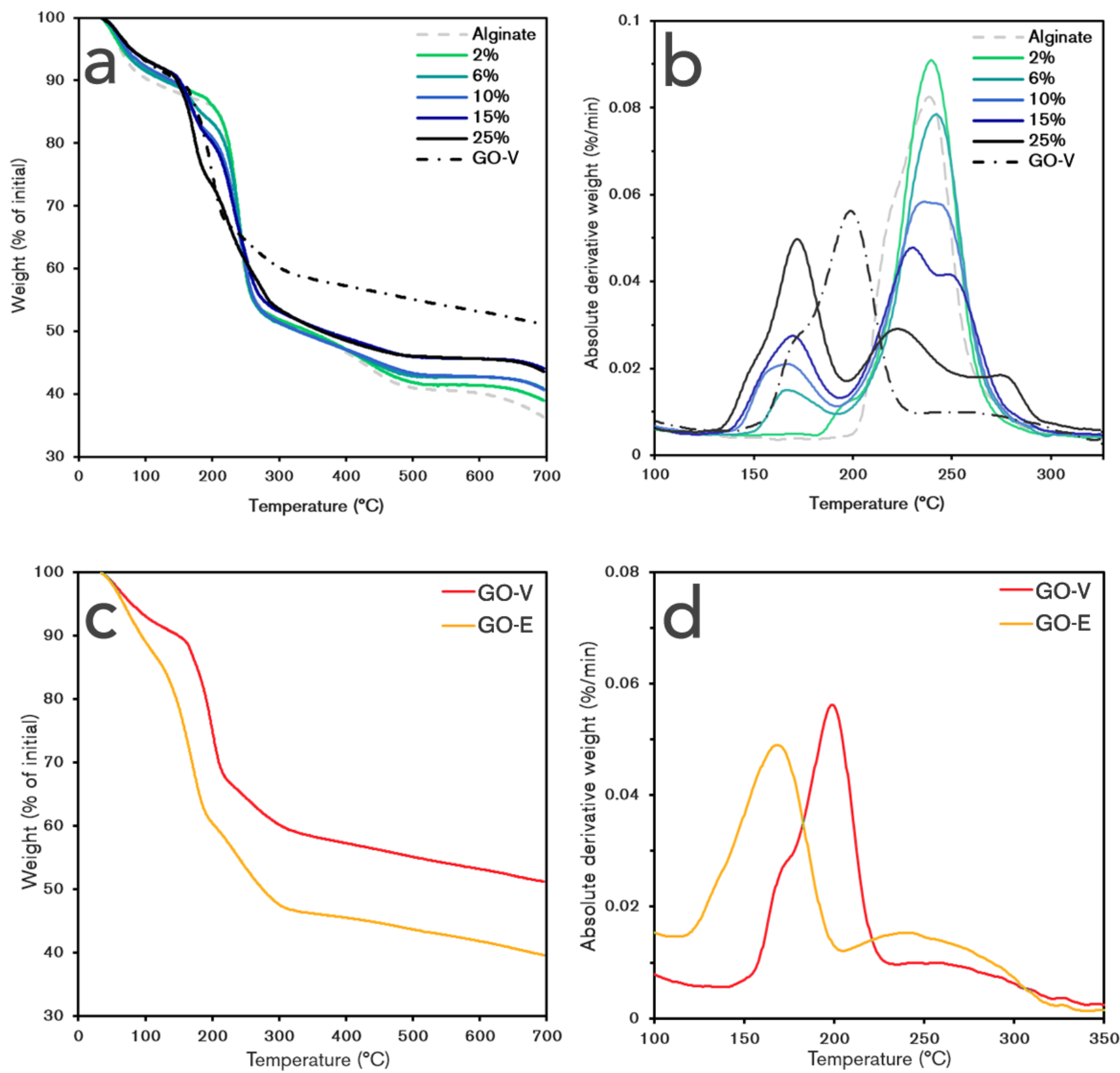

Figure 8-(a) Comparison of TGA thermograms: as GO content increases, the degradation rate decreases and thermal stability is improved. (b) The DTG curves illustrate this effect as the primary peak broadens and splits; further, a secondary peak appears, associated with trapped water. (c) TGA and (d) DTG curves comparing evaporation-cast (GO-E) and vacuum-filtered GO films (GO-V) demonstrate the impact of the assembly method on the moisture intercalation between $G O$ sheets in the nanocomposites. 
The more rapid initial degradation is reflected in the DTG by the emergence of a secondary peak from $125-200^{\circ} \mathrm{C}$ above $6 \mathrm{wt} \% \mathrm{GO}$; the loss of mass in this region is attributed to evaporation of trapped water, with the high GO content films trapping more moisture. To substantiate this, and to buttress the previous indications of trapped water from mechanical and spectroscopic analyses, TGA was also performed on a crumbled evaporation-cast GO film containing no alginate. There is a stark contrast between the degradation curves of GO assembled by the two different methods, suggesting that the more time-consuming vacuum assembly method could be more effective in expelling trapped moisture. The decomposition of the evaporation-cast GO control film (Figure 8c) closely resembles those of the cast composite films, with a strong dehydration-associated degradation peak occurring at lower temperatures (Figure 8d), and greater total mass loss, indicating higher moisture content.

Following these observations, a $6 \%$ film was heat-treated at $120^{\circ} \mathrm{C}$ for $24 \mathrm{~h}$. Though this sample was too brittle for mechanical analysis, FTIR and TGA were performed and the data compared with those for the as-prepared films. FTIR (Figure S5) shows a marked decrease in the intensity of the OH stretching band, as well as a rightward shift. This suggests a large amount of the trapped moisture is expelled during heat treatment, and the remaining moisture is more tightly constrained in the galleries. TGA (Figure S6) further illustrates that this moisture was indeed responsible for the mass loss observed below $200^{\circ} \mathrm{C}$ in the untreated films. Together, these experiments confirmed the presence of significant trapped moisture, and the potential of heat treatment to control the moisture content. 


\section{Moisture permeability tests}

A major area of interest in the development of synthetic nacre-like composites is their application in packaging for perishable foods. As the permeability of a polymer film to gases and moisture depends on the diffusion of molecules through the polymer matrix, the presence of impermeable nanobarriers increases the length of the diffusion path by redirecting escaping gases around them, increasing the effective film thickness (Figure S1). ${ }^{31}$ Thus, thin nacre-like films which present highly tortuous paths for gas diffusion could effectively replace multi-layer polymers that are currently used for their high barrier performance.

GO nanosheets, having excellent antibacterial activity and minimal cytotoxicity, have already attracted interest for food packaging applications. ${ }^{56,57}$ Furthermore, the enhancement of oxygen barrier properties in homogeneous polymer- and biopolymer-GO films has already been demonstrated. For example, Kim et al. reported up to $80 \%$ reduction in oxygen permeability in PVA-coated PET when $0.3 \mathrm{wt} \%$ GO was incorporated, and Unalan et al. reported a similar 79\% reduction when $0.3 \mathrm{wt} \%$ GO was added to pullulan biopolymer. ${ }^{58,59}$ Alginate, being food-safe and biodegradable, is an attractive biopolymer for food packaging; however, its poor water resistance precludes its standalone use in many such applications. ${ }^{60}$ Therefore, demonstrating that ultralarge GO can also effectively enhance moisture resistance of biopolymers such as alginate would further improve the potential of GO in creating biodegradable single-layer materials suitable for food packaging. 


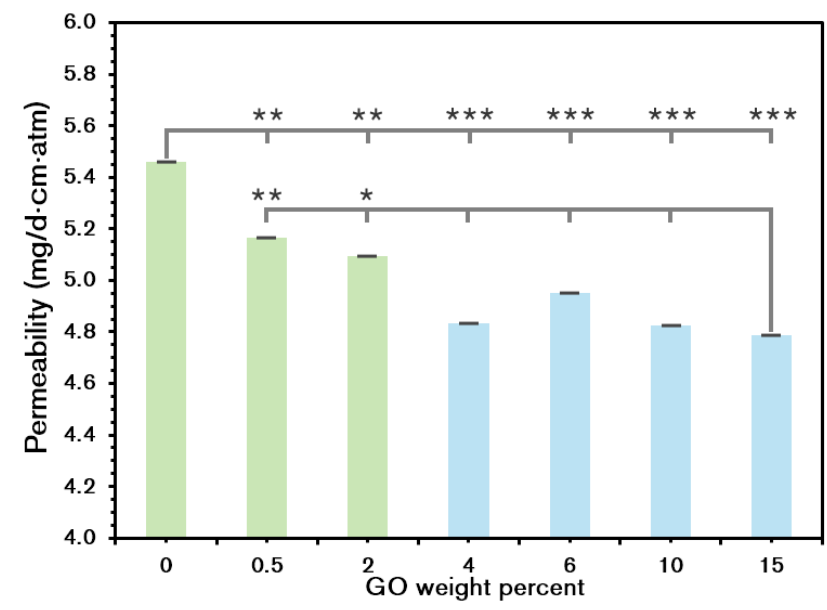

Figure 9-Permeability comparison of alginate and alginate-GO nanocomposite films. $(*: p<0.10 ; * *: p<$ $0.05 ; * * *: 0.01)$

To evaluate the improvement in barrier performance from the addition of ultralarge GO, a longterm moisture permeability experiment was conducted. Figure S7 illustrates the loss of water vapour through the nanocomposite films over one month. The slope of this graph normalised by the area of the aperture through which moisture could escape represents the measured water vapour transmission rate, $\mathrm{WVTR}_{\mathrm{M}}$. As the $\mathrm{WVTR}_{\mathrm{M}}$ is high, it is necessary to correct for the resistance of the stagnant air layer between the water surface and the film ${ }^{61}$ :

$$
\begin{gathered}
W V T R_{C}=\eta \cdot W V T R_{M} \\
\eta=\frac{P_{V}\left(R H_{I N}-R H_{O U T}\right)}{\left(P_{C}-P_{V} R H_{O U T}\right)}
\end{gathered}
$$

where $\eta$ is the correction factor, $P_{V}$ is the saturation vapour pressure of water at the experimental temperature (therefore multiplied by the relative humidity to represent the partial pressure of water), and $P_{C}$ is the partial pressure of water at the boundary of the air layer and the film, calculated according to the method of Gennadios et al. ${ }^{61} P_{C}$ is dependent on the distance of the film to the water surface; here, since $\mathrm{WVTR}_{\mathrm{M}}$ remained constant throughout the experiment, a conservative constant gap of 4 $\mathrm{cm}$ was assumed. Finally, the relative humidity at the surface of the water was assumed to be $100 \%$ 
(saturated), and the relative humidity of the air outside the tubes was maintained at $40 \%$. To facilitate comparison, the corrected $\mathrm{WVTR}_{\mathrm{C}}$ was further converted to water vapour permeability (WVP), where $d(0.04 \mathrm{~mm})$ is the film thickness:

$$
W V P=\frac{W V T R_{C}}{P_{V}(\Delta R H)} \cdot d
$$

The permeability data are presented in Figure 9 and tabulated in Table S3. The neat alginate film is more permeable to moisture than any of the alginate-GO nanocomposites, with a WVP of 5.46 $\mathrm{mg} / \mathrm{d} \cdot \mathrm{cm} \cdot \mathrm{atm}$. This measured WVP agrees well with an earlier report that used the same alginate source, and measured $5.57 \mathrm{mg} / \mathrm{d} \cdot \mathrm{cm} \cdot \mathrm{atm}^{62}$ As the GO content increases, the resistance to moisture penetration steadily improves, ranging from $5.16 \mathrm{mg} / \mathrm{d} \cdot \mathrm{cm} \cdot \mathrm{atm}$ to $4.79 \mathrm{mg} / \mathrm{d} \cdot \mathrm{cm} \cdot \mathrm{atm}$ as $\mathrm{GO}$ content increased from 0.5 to $15 \mathrm{wt} \%$, representing over $12 \%$ improvement over the alginate control. These results represent a statistically significant enhancement in barrier performance consistent with the improvements tabulated by Duncan in his 2011 review $^{31}$, and corroborate the TGA observations of improved moisture retention. Hence, ultralarge GO can indeed form effective diffusion barriers, suggesting a compelling avenue for further development towards effective packaging materials for fresh foods.

The intercalation of water into the layered composites suggests that some of the evaporating moisture can also be retained in the films. Thus, the ultralarge GO which reinforces the nanocomposite can affect the moisture barrier properties by simultaneously containing moisture within the film and hindering its transport through it. By modifying the assembly process — for example, crosslinking the alginate ${ }^{17,23,57}$, adjusting the GO concentration, or using rGO to further 
improve gas and moisture barrier performance ${ }^{12,58}$ — the balance of these properties could be finetuned in future studies for specific applications.

Finally, the relative moisture retention performance of the composite films exhibits an interesting clustering behaviour: although all of the composite films are significantly less permeable than the alginate control, those containing $2 \mathrm{wt} \% \mathrm{GO}$ and lower also have significantly higher permeability than those with $4 \%$ and above. Across $4 \%$ to $15 \%$, the permeability of the composites does not differ significantly (Figure 9). This observation mirrors the observed enhancements of mechanical properties and thermal stability above a self-assembly threshold of $2 \%$, and again corresponds to the observed formation of ultralarge GO LC domains in suspension.

\section{Conclusions}

The facile, bioinspired assembly of ultralarge GO sheets in alginate was demonstrated using a one-step evaporation casting method. A novel method was used to observe the alginate-GO system through the entire casting process. In aqueous suspension with alginate, ultralarge GO self-assembled into lyotropic nematic LC domains above $2 \mathrm{wt} \%$. Upon evaporation, LC alginate-GO suspensions yielded dense, highly-ordered layered structures which were directly observed with SEM. However, low GO suspensions exhibited no LC formation, and their cast films revealed a mixture of aligned and randomly-oriented domains. The importance of GO content in determining LC formation and the composite nanostructure was therefore established.

The mechanical properties also reflected the self-assembly of GO. The cast nanocomposite films with greater than $2 \mathrm{wt} \% \mathrm{GO}$ content demonstrated remarkable improvements in elastic modulus, closely matching theoretical models for platelet-reinforced nanocomposites with random and parallel 
orientations. Up to $230 \%$ improvement in elastic modulus was achieved at $15 \mathrm{wt} \% \mathrm{GO}(12.59 \pm 0.19$ $\mathrm{GPa}$ ), representing one of the highest values measured for biopolymer-GO composites. The evolution of the ultimate strength and toughness of the composite films suggested the inclusion of defects in the microstructure as GO loading increased.

Spectroscopic studies of the cast films revealed the presence of hydrogen bonding, and the absence of covalent interactions, between the alginate and the ultralarge GO. Together with thermogravimetric analysis, these observations shed light on the DMA measurements, suggesting high amounts of water intercalation into the layered composite microstructure as a result of the evaporation casting process. TGA also demonstrated the stabilizing effect of the ultralarge GO on the polymer, significantly delaying its thermal degradation. Finally, the brick-and-mortar structure formed by the ultralarge GO sheets served as an effective barrier to moisture transport. The simultaneous hindrance of diffusion through the films and retention of moisture within them presents an attractive opportunity for designing films fine-tuned for specific packaging requirements.

This investigation observed alginate-GO composites through the entire assembly process, studying LC formation in suspension and its effects on the structure and performance of the cast nanocomposites. The enhancement of each of the mechanical, thermal, and barrier performances of the films was a direct result of the homogeneous, layered microstructure formed only upon the evaporation of LC alginate-GO dispersions. The insights gained by this study will therefore help guide the rational design of versatile, robust, and biodegradable nacre-like nanocomposites as this technology continues to mature. 


\section{Acknowledgements}

The authors acknowledge the Canada Research Chairs Program, the Canada Foundation for Innovation, the Natural Sciences and Engineering Research Council of Canada (NSERC/CRSNG), the Fonds de recherche du Québec — Nature et technologies (FRQNT), and McGill University, for their financial support. SXW was supported by an NSERC Undergraduate Student Research Award (USRA), an FRQNT Master's Research Scholarship, a Graduate Excellence Fellowship (Faculty of Engineering), and the Eugenie Ulmer Lamothe Fund (Department of Chemical Engineering).

The FTIR and TG-DSC were performed at the Centre for Self-Assembled Chemical Structures (CSACS/CRMAA), part of the McGill Chemistry Material Characterization $\left(\mathrm{MC}^{2}\right)$ facility. The authors acknowledge the $\mathrm{MC}^{2}$ staff for their technical support and the use of their equipment. The operation of $\mathrm{MC}^{2}$ is supported by the Québec Center for Advanced Materials (QCAM/CQMF). SEM studies were performed at the McGill Institute for Advanced Materials (MIAM) and Materials Engineering Electron Microscopy Labs, respectively.

The authors are grateful to L. Reven (McGill University) for the use of the POM. The authors also thank M. Cerruti (McGill University) and P. Hubert (McGill University) for the use of the Raman microscope and DMA, respectively. 


\section{Disclosure}

The authors of this study report no conflicts of interest relevant to this article.

\section{Supporting Information}

A comprehensive Supporting Information document is provided, which includes: supplementary images, tabulated mechanical and moisture permeability data, Raman spectra, FTIR spectra assignments, experiments on the effect of thermal annealing and the comparison of assembly methods, details of image editing techniques, and the corresponding original images.

\section{Graphical Summary}

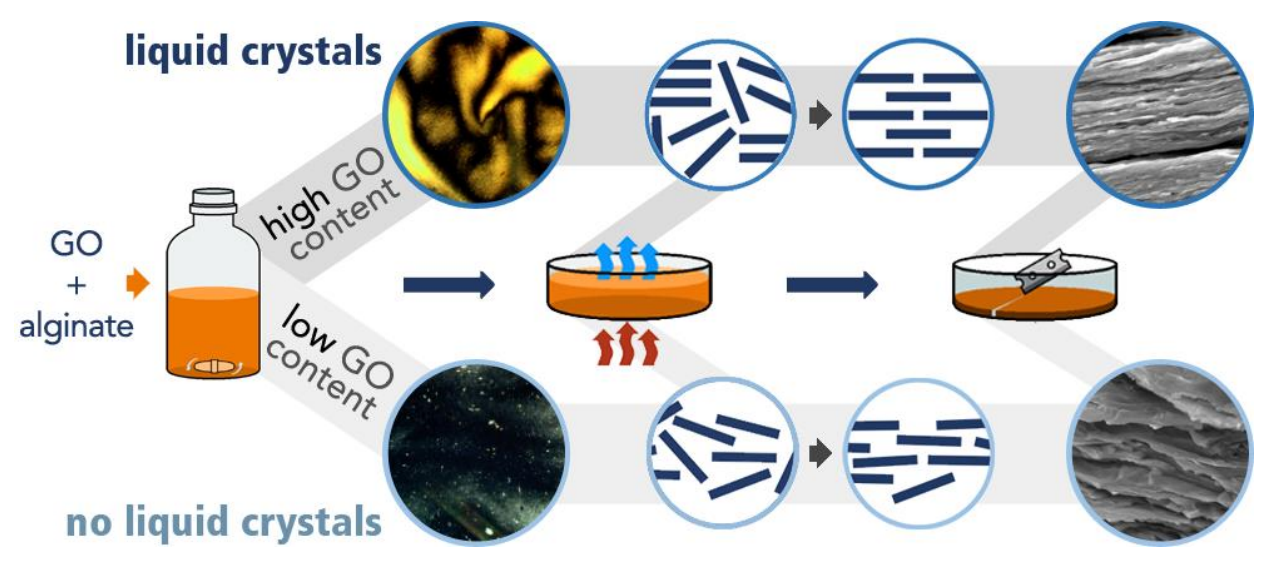




\section{References}

1. Aboutalebi, S. H., Gudarzi, M. M., Zheng, Q. B. \& Kim, J.-K. (2011). Spontaneous Formation of Liquid Crystals in Ultralarge Graphene Oxide Dispersions. Adv. Funct. Mater. 21, 2978-2988

2. Zhang, Y., Gong, S., Zhang, Q., Ming, P., Wan, S., Peng, J., Jiang, L. \& Cheng, Q. (2016). Graphene-based artificial nacre nanocomposites. Chem. Soc. Rev. 45, 2378-2395

3. Kim, J. E., Han, T. H., Lee, S. H., Kim, J. Y., Ahn, C. W., Yun, J. M. \& Kim, S. O. (2011). Graphene Oxide Liquid Crystals. Angew. Chem., Int. Ed. 50, 3043-3047

4. Dan, B., Behabtu, N., Martinez, A., Evans, J. S., Kosynkin, D. V., Tour, J. M., Pasquali, M. \& Smalyukh, I. I. (2011). Liquid crystals of aqueous, giant graphene oxide flakes. Soft Matter 7, 11154-11159

5. Xu, Z. \& Gao, C. (2011). Aqueous Liquid Crystals of Graphene Oxide. ACS Nano 5, 2908-2915

6. Frenkel, D. (1989). Columnar ordering as an excluded-volume effect. (Invited Lecture). Liq. Cryst. 5, 929-940

7. Mejia, A. F., Chang, Y.-W., Ng, R., Shuai, M., Mannan, M. S. \& Cheng, Z. (2012). Aspect ratio and polydispersity dependence of isotropic-nematic transition in discotic suspensions. Phys. Rev. E 85, 061708

8. Yousefi, N., Gudarzi, M. M., Zheng, Q., Hamed Aboutalebi, S., Sharif, F. \& Kim, J.-K. (2012). Self-alignment and high electrical conductivity of ultralarge graphene oxide-polyurethane nanocomposites. J. Mater. Chem. 22, 12709-12717

9. Yousefi, N., Lin, X., Zheng, Q., Shen, X., Pothnis, J. R., Jia, J., Zussman, E. \& Kim, J.-K. (2013). Simultaneous in situ reduction, self-alignment and covalent bonding in graphene oxide/epoxy composites. Carbon 59, 406-417

10. Jalili, R., Aboutalebi, S. H., Esrafilzadeh, D., Konstantinov, K., Moulton, S. E., Razal, J. M. \& Wallace, G. G. (2013). Organic Solvent-Based Graphene Oxide Liquid Crystals: A Facile Route toward the Next Generation of Self-Assembled Layer-by-Layer Multifunctional 3D Architectures. ACS Nano 7, 3981-3990

11. Cheng, Q., Duan, J., Zhang, Q. \& Jiang, L. (2015). Learning from Nature: Constructing Integrated Graphene-Based Artificial Nacre. ACS Nano 9, 2231-2234 
12. Yousefi, N., Gudarzi, M. M., Zheng, Q., Lin, X., Shen, X., Jia, J., Sharif, F. \& Kim, J.-K. (2013). Highly aligned, ultralarge-size reduced graphene oxide/polyurethane nanocomposites: Mechanical properties and moisture permeability. Composites, Part A. 49, 42-50

13. Wang, J., Cheng, Q. \& Tang, Z. (2012). Layered nanocomposites inspired by the structure and mechanical properties of nacre. Chem. Soc. Rev. 41, 1111-1129

14. Wan, S., Peng, J., Li, Y., Hu, H., Jiang, L. \& Cheng, Q. (2015). Use of Synergistic Interactions to Fabricate Strong, Tough, and Conductive Artificial Nacre Based on Graphene Oxide and Chitosan. ACS Nano 9, 9830-9836

15. Lee, W., Lee, J. U., Jung, B. M., Byun, J.-H., Yi, J.-W., Lee, S.-B. \& Kim, B.-S. (2013). Simultaneous enhancement of mechanical, electrical and thermal properties of graphene oxide paper by embedding dopamine. Carbon 65, 296-304

16. Zhong, D., Yang, Q., Guo, L., Dou, S., Liu, K. \& Jiang, L. (2013). Fusion of nacre, mussel, and lotus leaf: bio-inspired graphene composite paper with multifunctional integration. Nanoscale 5, $5758-5764$

17. Cui, W., Li, M., Liu, J., Wang, B., Zhang, C., Jiang, L. \& Cheng, Q. (2014). A Strong Integrated Strength and Toughness Artificial Nacre Based on Dopamine Cross-Linked Graphene Oxide. ACS Nano 8, 9511-9517

18. Ionita, M., Pandele, M. A. \& Iovu, H. (2013). Sodium alginate/graphene oxide composite films with enhanced thermal and mechanical properties. Carbohydr. Polym. 94, 339-344

19. Cao, K., Jiang, Z., Zhao, J., Zhao, C., Gao, C., Pan, F., Wang, B., Cao, X. \& Yang, J. (2014). Enhanced water permeation through sodium alginate membranes by incorporating graphene oxides. J. Membr. Sci. 469, 272-283

20. Chen, K., Shi, B., Yue, Y., Qi, J. \& Guo, L. (2015). Binary Synergy Strengthening and Toughening of Bio-Inspired Nacre-like Graphene Oxide/Sodium Alginate Composite Paper. ACS Nano 9, 8165-8175

21. Nie, L., Liu, C., Wang, J., Shuai, Y., Cui, X. \& Liu, L. (2015). Effects of surface functionalized graphene oxide on the behavior of sodium alginate. Carbohydr. Polym. 117, 616-623

22. Vilcinskas, K., Norder, B., Goubitz, K., Mulder, F. M., Koper, G. J. M. \& Picken, S. J. (2015). Tunable Order in Alginate/Graphene Biopolymer Nanocomposites. Macromolecules 48, 83238330 
23. Zheng, H., Yang, J. \& Han, S. (2016). The synthesis and characteristics of sodium alginate/graphene oxide composite films crosslinked with multivalent cations. J. Appl. Polym. Sci. 133

24. Meyers, M. A., Chen, P.-Y., Lin, A. Y.-M. \& Seki, Y. (2008). Biological materials: Structure and mechanical properties. Prog. Mater. Sci. 53, 1-206

25. Wegst, U. G. K., Bai, H., Saiz, E., Tomsia, A. P. \& Ritchie, R. O. (2015). Bioinspired structural materials. Nat. Mater. 14, 23

26. Liu, A., Walther, A., Ikkala, O., Belova, L. \& Berglund, L. A. (2011). Clay Nanopaper with Tough Cellulose Nanofiber Matrix for Fire Retardancy and Gas Barrier Functions. Biomacromolecules 12, 633-641

27. Aulin, C., Salazar-Alvarez, G. \& Lindström, T. (2012). High strength, flexible and transparent nanofibrillated cellulose-nanoclay biohybrid films with tunable oxygen and water vapor permeability. Nanoscale 4, 6622-6628

28. Wu, C.-N., Saito, T., Fujisawa, S., Fukuzumi, H. \& Isogai, A. (2012). Ultrastrong and High GasBarrier Nanocellulose/Clay-Layered Composites. Biomacromolecules 13, 1927-1932

29. Sehaqui, H., Kochumalayil, J., Liu, A., Zimmermann, T. \& Berglund, L. A. (2013). Multifunctional Nanoclay Hybrids of High Toughness, Thermal, and Barrier Performances. ACS Appl. Mater. Interfaces 5, 7613-7620

30. Kochumalayil, J. J., Bergenstråhle-Wohlert, M., Utsel, S., Wågberg, L., Zhou, Q. \& Berglund, L. A. (2013). Bioinspired and Highly Oriented Clay Nanocomposites with a Xyloglucan Biopolymer Matrix: Extending the Range of Mechanical and Barrier Properties. Biomacromolecules 14, 84-91

31. Duncan, T. V. (2011). Applications of nanotechnology in food packaging and food safety: Barrier materials, antimicrobials and sensors. J. Colloid Interface Sci. 363, 1-24

32. Hummers, W. S. \& Offeman, R. E. (1958). Preparation of Graphitic Oxide. J. Am. Chem. Soc. 80, 1339-1339

33. Geng, Y., Wang, S. J. \& Kim, J.-K. (2009). Preparation of graphite nanoplatelets and graphene sheets. J. Colloid Interface Sci. 336, 592-598

34. Yousefi, N., W. Wong, K. K., Hosseinidoust, Z., Osholm Sørensen, H., Bruns, S., Zheng, Y. \& Tufenkji, N. (2018). Hierarchically porous, ultra-strong reduced graphene oxide-cellulose nanocrystal sponges for exceptional adsorption of water contaminants. Nanoscale 10, 7171-7184 
35. Halpin, J. C. \& Kardos, J. L. The Halpin-Tsai equations: A review. Polym. Eng. Sci. 16, 344-352

36. van Es, M. (2001). Polymer-clay nanocomposites: the importance of particle dimensions. Ph.D. Dissertation, Technische Universiteit Delft, Delft NL.

37. Kinloch, A. J. \& Taylor, A. C. (2003). Mechanical and fracture properties of epoxy/inorganic micro- and nano-composites. J. Mater. Sci. Lett. 22, 1439-1441

38. Liang, J., Huang, Y., Zhang, L., Wang, Y., Ma, Y., Guo, T. \& Chen, Y. (2009). Molecular-Level Dispersion of Graphene into Poly(vinyl alcohol) and Effective Reinforcement of their Nanocomposites. Adv. Funct. Mater. 19, 2297-2302

39. Suk, J. W., Piner, R. D., An, J. \& Ruoff, R. S. (2010). Mechanical Properties of Monolayer Graphene Oxide. ACS Nano 4, 6557-6564

40. Compton, O. C., Cranford, S. W., Putz, K. W., An, Z., Brinson, L. C., Buehler, M. J. \& Nguyen, S. T. (2012). Tuning the Mechanical Properties of Graphene Oxide Paper and Its Associated Polymer Nanocomposites by Controlling Cooperative Intersheet Hydrogen Bonding. ACS Nano 6, 2008-2019

41. Gómez-Navarro, C., Weitz, R. T., Bittner, A. M., Scolari, M., Mews, A., Burghard, M. \& Kern, K. (2007). Electronic Transport Properties of Individual Chemically Reduced Graphene Oxide Sheets. Nano Lett. 7, 3499-3503

42. Kudin, K. N., Ozbas, B., Schniepp, H. C., Prud'homme, R. K., Aksay, I. A. \& Car, R. (2008). Raman Spectra of Graphite Oxide and Functionalized Graphene Sheets. Nano Lett. 8, 36-41

43. Pan, D., Wang, S., Zhao, B., Wu, M., Zhang, H., Wang, Y. \& Jiao, Z. (2009). Li Storage Properties of Disordered Graphene Nanosheets. Chem. Mater. 21, 3136-3142

44. Marcano, D. C., Kosynkin, D. V., Berlin, J. M., Sinitskii, A., Sun, Z., Slesarev, A., Alemany, L. B., Lu, W. \& Tour, J. M. (2010). Improved Synthesis of Graphene Oxide. ACS Nano 4, 48064814

45. Ferrari, A. C., Meyer, J. C., Scardaci, V., Casiraghi, C., Lazzeri, M., Mauri, F., Piscanec, S., Jiang, D., Novoselov, K. S., Roth, S. \& Geim, A. K. (2006). Raman Spectrum of Graphene and Graphene Layers. Phys. Rev. Lett. 97, 187401

46. Chen, J.-H., Cullen, W. G., Jang, C., Fuhrer, M. S. \& Williams, E. D. (2009). Defect Scattering in Graphene. Phys. Rev. Lett. 102, 236805 
47. Fang, M., Wang, K., Lu, H., Yang, Y. \& Nutt, S. (2009). Covalent polymer functionalization of graphene nanosheets and mechanical properties of composites. J. Mater. Chem. 19, 7098-7105

48. Titelman, G. I., Gelman, V., Bron, S., Khalfin, R. L., Cohen, Y. \& Bianco-Peled, H. (2005). Characteristics and microstructure of aqueous colloidal dispersions of graphite oxide. Carbon 43, 641-649

49. Lawrie, G., Keen, I., Drew, B., Chandler-Temple, A., Rintoul, L., Fredericks, P. \& Grøndahl, L. (2007). Interactions between Alginate and Chitosan Biopolymers Characterized Using FTIR and XPS. Biomacromolecules 8, 2533-2541

50. Acik, M., Lee, G., Mattevi, C., Pirkle, A., Wallace, R. M., Chhowalla, M., Cho, K. \& Chabal, Y. (2011). The Role of Oxygen during Thermal Reduction of Graphene Oxide Studied by Infrared Absorption Spectroscopy. J. Phys. Chem. C 115, 19761-19781

51. Verma, D., Katti, K. \& Katti, D. (2007). Nature of water in nacre: A 2D Fourier transform infrared spectroscopic study. Spectrochim. Acta, Part A. 67, 784-788

52. Chen, X., Liu, G., Zhang, H. \& Fan, Y. (2015). Fabrication of graphene oxide composite membranes and their application for pervaporation dehydration of butanol. Chin. J. Chem. Eng. 23, 1102-1109

53. Gorman, M. (1957). The evidence from infrared spectroscopy for hydrogen bonding: A case history of the correlation and interpretation of data. J. Chem. Educ. 34, 304

54. Nibbering, E. T. J., Dreyer, J., Kühn, O., Bredenbeck, J., Hamm, P. \& Elsaesser, T. (2007). Vibrational dynamics of hydrogen bonds. Analysis and Control of Ultrafast Photoinduced Reactions 619-687

55. Soares, J. P., Santos, J. E., Chierice, G. O. \& Cavalheiro, E. T. G. (2004). Thermal behavior of alginic acid and its sodium salt. Eclética Quim. 29, 57-64

56. Hu, W., Peng, C., Luo, W., Lü, M., Li, X., Li, D., Huang, Q. \& Fan, C. (2010). GrapheneBased Antibacterial Paper. ACS Nano 4, 4317-4323

57. Grande, C. D., Mangadlao, J., Fan, J., Leon, A. D., Delgado-Ospina, J., Rojas, J. G., Rodrigues, D. F. \& Advincula, R. (2017). Chitosan Cross-Linked Graphene Oxide Nanocomposite Films with Antimicrobial Activity for Application in Food Industry. Macromol. Symp. 374, 1600114

58. Kim, H. M., Lee, J. K. \& Lee, H. S. (2011). Transparent and high gas barrier films based on poly(vinyl alcohol)/graphene oxide composites. Thin Solid Films 519, 7766-7771 
59. Unalan, I. U., Wan, C., Figiel, Łukasz, Olsson, R. T., Trabattoni, S. \& Farris, S. (2015). Exceptional oxygen barrier performance of pullulan nanocomposites with ultra-low loading of graphene oxide. Nanotechnology 26, 275703

60. Rhim, J.-W. (2004). Physical and mechanical properties of water resistant sodium alginate films. LWT_Food Sci. Technol. 37, 323-330

61. Gennadios, A., Weller, C. L. \& Gooding, C. H. (1994). Measurement errors in water vapor permeability of highly permeable, hydrophilic edible films. J. Food Eng. 21, 395-409

62. Huq, T., Salmieri, S., Khan, A., Khan, R. A., Le Tien, C., Riedl, B., Fraschini, C., Bouchard, J., Uribe-Calderon, J., Kamal, M. R. \& Lacroix, M. (2012). Nanocrystalline cellulose (NCC) reinforced alginate based biodegradable nanocomposite film. Carbohydr. Polym. 90, 1757-1763 


\section{Supporting Information}

\section{LC formation in ultralarge GO}

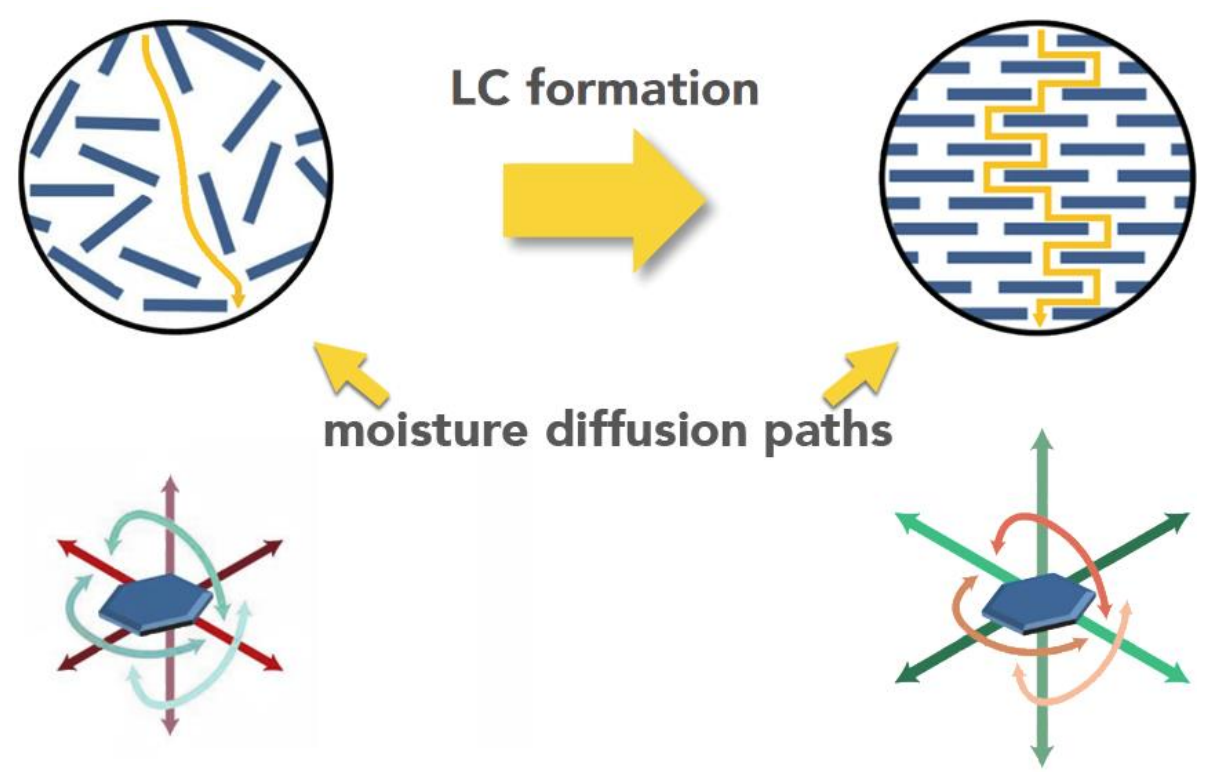

Figure S1 - LC alignment of highly-anisotropic GO sheets is driven by an excluded-volume effect. As rotational entropy is further constrained, translational entropy is increased. Cast films with highly-ordered structures form effective barriers by increasing the tortuosity of the diffusion path through the material.

Ultralarge graphene oxide

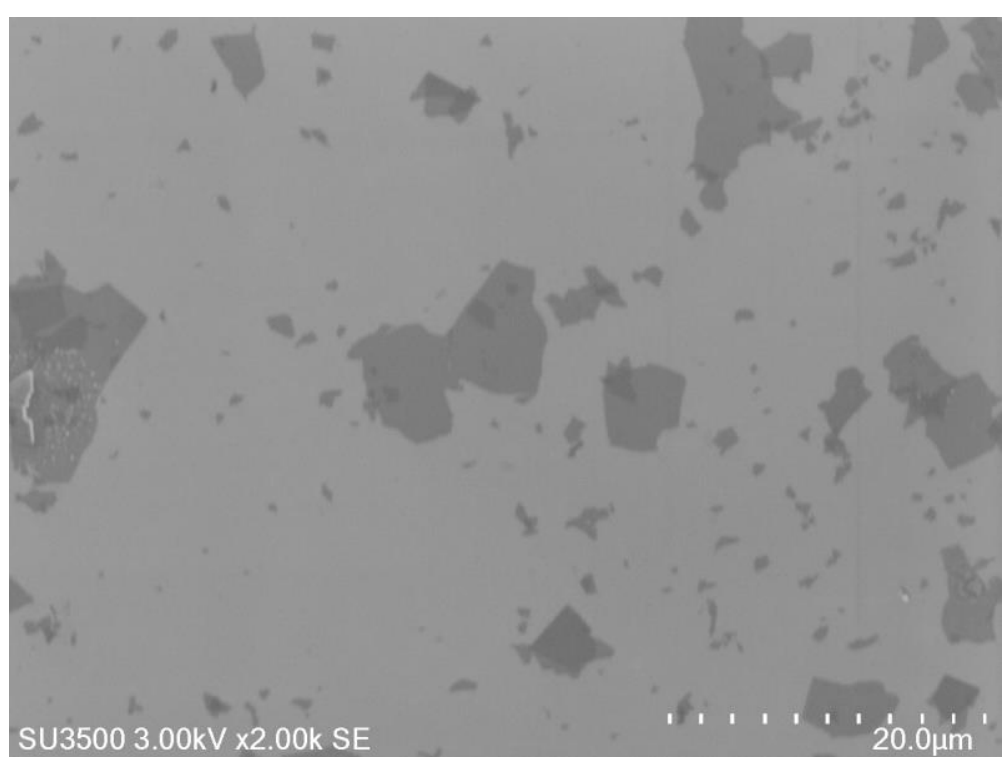

Figure S2 - Representative SEM micrograph of ultralarge GO processed using the modified Hummers method, deposited on a silica substrate. The average aspect ratio of $G O$ nanosheets processed using this method is approximately $1000 .^{1}$ 


\section{Supplementary SEM images}
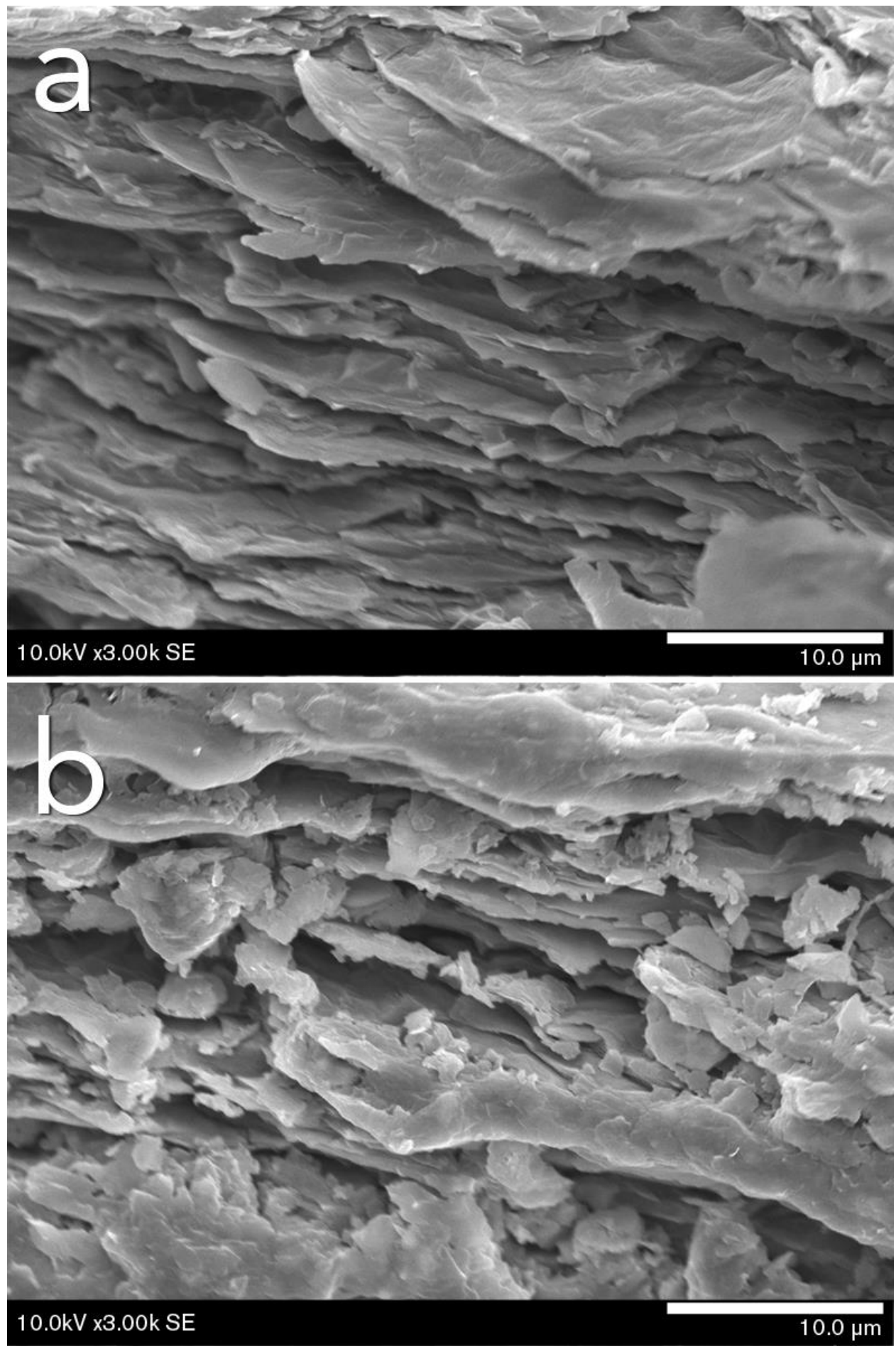

Figure $S_{3}-S E M$ comparison of (a) highly-aligned and (b) less-aligned domains in AG1. 


\section{Mechanical properties}

Table S1 - Comparison of mechanical properties GO weight percent Elastic modulus (GPa) Ultimate strength* Toughness** $\left(\mathrm{MJ} / \mathrm{m}^{3}\right)$ (MPa)

\begin{tabular}{crll}
\hline Alginate & $3.79 \pm 0.37$ & $71.4 \pm 6.4$ & $6.07 \pm 2.76$ \\
$\mathbf{0 . 5 \%}$ & $4.85 \pm 0.65$ & $65.5 \pm 7.1$ & $6.51 \pm 2.21$ \\
$\mathbf{1 \%}$ & $4.16 \pm 1.06$ & $74.0 \pm 7.4$ & $5.71 \pm 2.80$ \\
$\mathbf{2 \%}$ & $4.49 \pm 0.71$ & $74.0 \pm 6.4$ & $4.45 \pm 1.72$ \\
$\mathbf{3 \%}$ & $6.31 \pm 0.33$ & $69.4 \pm 12.7$ & $3.42 \pm 3.24$ \\
$\mathbf{4 \%}$ & $7.42 \pm 2.01$ & $59.3 \pm 16.3$ & $1.49 \pm 0.30$ \\
$\mathbf{6 \%}$ & $8.36 \pm 1.04$ & $85.3 \pm 9.9$ & $0.92 \pm 0.76$ \\
$\mathbf{1 0 \%}$ & $9.78 \pm 0.70$ & $71.8 \pm 19.3$ & $0.34 \pm 0.16$ \\
$\mathbf{1 5 \%}$ & $12.59 \pm 0.19$ & $43.7 \pm 16.0$ & $0.58 \pm 0.39$ \\
$\mathbf{2 5 \%}$ & $7.04 \pm 0.82$ & $29.8 \pm 2.6$ & $0.36 \pm 0.04$ \\
\hline
\end{tabular}

$\mu \pm \sigma ;{ }^{*}$ At fracture; ${ }^{* *}$ Toughness is measured by the area under stress-strain curve.

\section{Raman spectra}

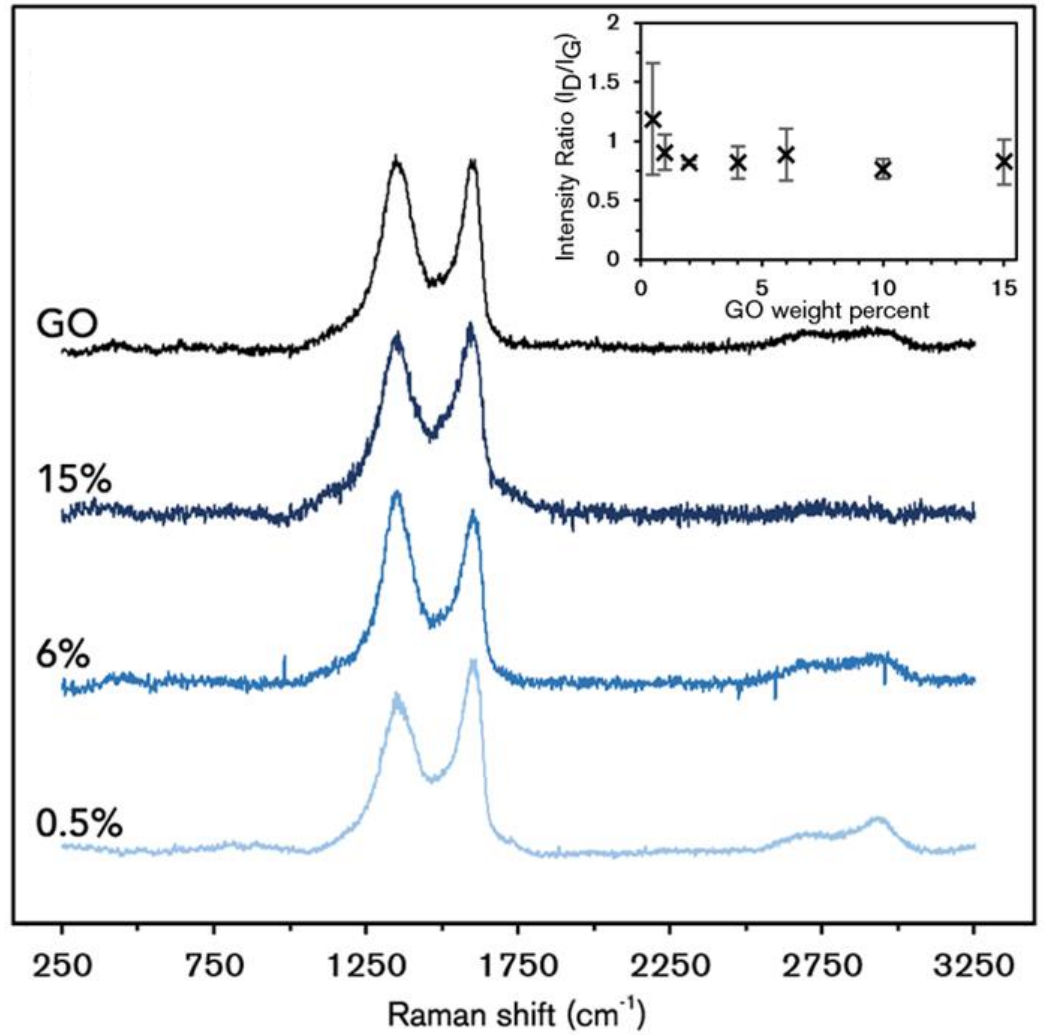

Figure $\mathrm{S}_{4}$ - Representative Raman spectra of alginate-GO nanocomposite films. Inset: average intensity ratio of observed $D$ and $G$ peaks in Raman spectra. 
FTIR spectra assignments

Table S2 - FTIR spectra assignments

\begin{tabular}{|c|c|c|}
\hline \multicolumn{2}{|c|}{ Wavenumber $\left(\mathrm{cm}^{-1}\right)$} & \multirow{2}{*}{ Assignment } \\
\hline Alginate & GO & \\
\hline 3266 (broad) & 3329 (broad) & $\mathrm{OH}$ stretch \\
\hline \multicolumn{2}{|c|}{$3050-2850$} & trapped water \\
\hline \multicolumn{2}{|c|}{2900} & $\mathrm{C}-\mathrm{H}$ stretch \\
\hline - & 1738 & $C=O$ stretch \\
\hline - & 1611 & $C=C$ stretch \\
\hline 1600 & - & symmetric $\mathrm{COO}^{-}$stretch \\
\hline 1410 & - & $\begin{array}{c}\text { asymmetric } \mathrm{COO}^{-} \\
\text {stretch }\end{array}$ \\
\hline - & 1360 & C-O (carboxyl) \\
\hline- & 1240 & C-O (epoxide) \\
\hline 1025 & 1040 & $\mathrm{C}-\mathrm{O}$ \\
\hline
\end{tabular}

Effects of thermal annealing

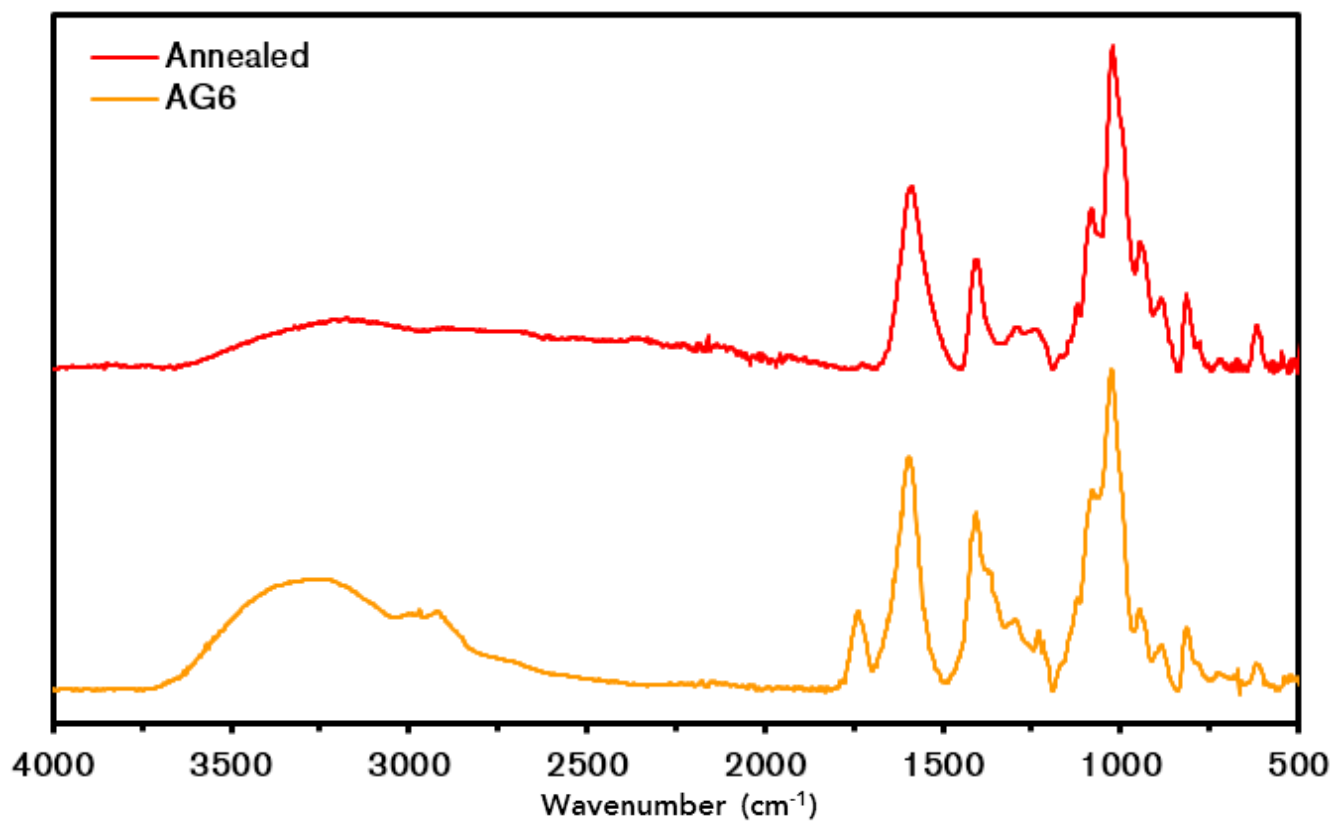

Figure $S_{5}-F T I R$ comparison of as-prepared film (6 wt\% GO) with film annealed at $120^{\circ} \mathrm{C}$ for $24 \mathrm{~h}$.

Comparing the as-prepared $6 \%$ film before and after $24 \mathrm{~h}$ heat treatment at $120^{\circ} \mathrm{C}$, a marked rightward shift and decrease is observed in the $\mathrm{OH}$ stretching band as trapped moisture is evaporated, and remaining moisture becomes more constrained. The weakening of the $\mathrm{C}=\mathrm{O}$ and $\mathrm{C}-\mathrm{O}$ stretching 
bands (1740 and $1360 \mathrm{~cm}^{-1}$ respectively) suggests that some GO reduction occurred during heat treatment.

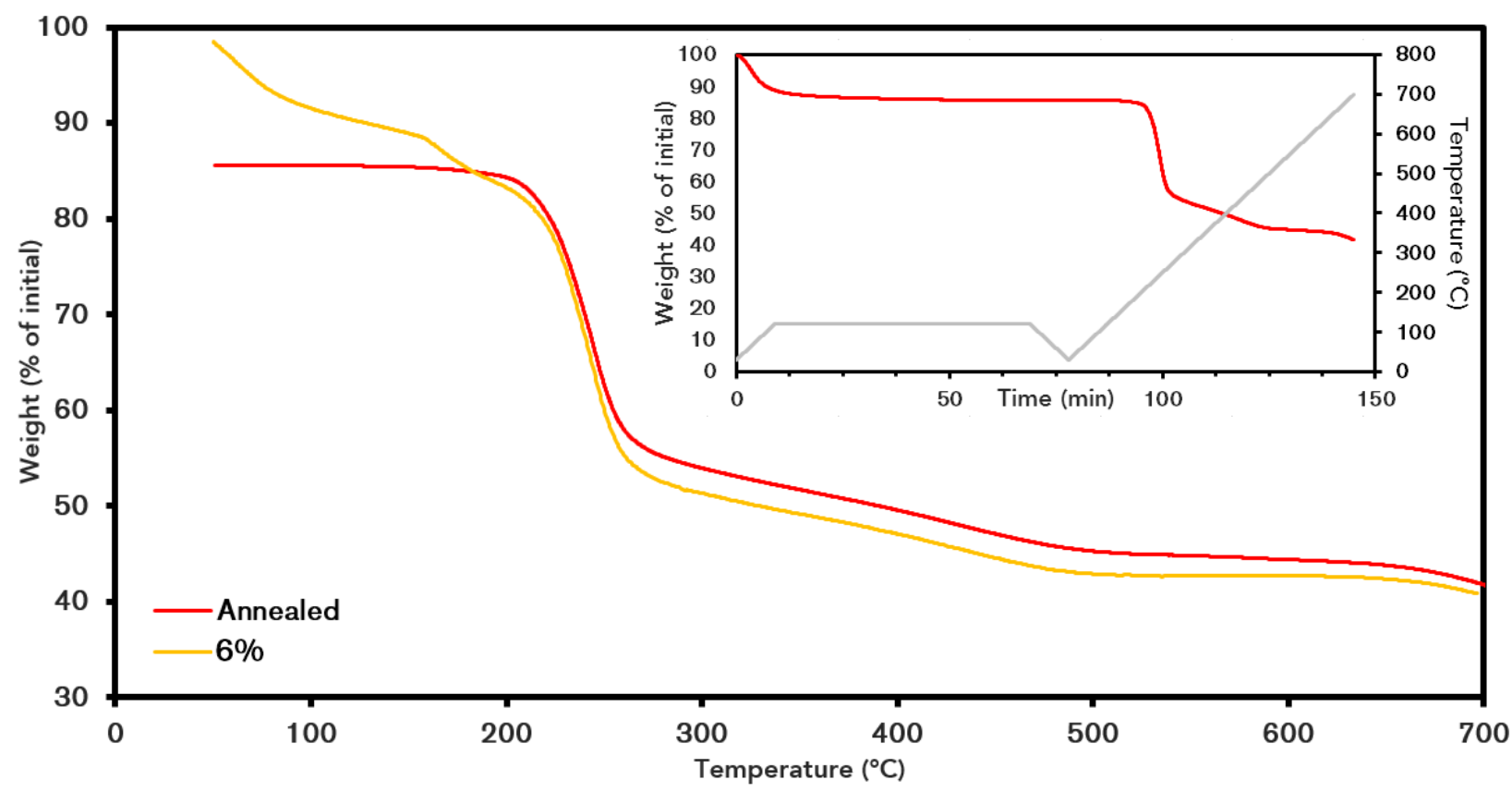

Figure S6 - TGA comparison of as-prepared film (6 wt\% GO) with film annealed at $120^{\circ} \mathrm{C}$ for $24 \mathrm{~h}$. Inset: mass (red) and temperature profile (grey) during preheating of annealed sample

Several weeks passed before TGA could be performed on the heat-treated film; therefore, a preheating step of $1 \mathrm{~h}$ at $120^{\circ} \mathrm{C}$, under $\mathrm{N}_{2}$ purge, was performed for this sample before performing the standard $30-700^{\circ} \mathrm{C}$ ramp experiment. Returning the sample to the original temperature of the heat treatment would ensure any moisture absorbed during storage was expelled before the experiment, and better facilitate comparison with the untreated film.

The significant moisture content of the untreated nanocomposites is illustrated upon comparing the as-prepared $6 \%$ film before and after heat treatment. Treatment at $120^{\circ} \mathrm{C}$ removed the moisture that was responsible for the loss of mass below $\sim 200^{\circ} \mathrm{C}$ in the untreated samples. The residual mass is higher throughout the experiment; however, it is clear that a large amount of the moisture removed during the initial $24 \mathrm{~h}$ treatment was re-absorbed during prolonged storage. 
Moisture permeability

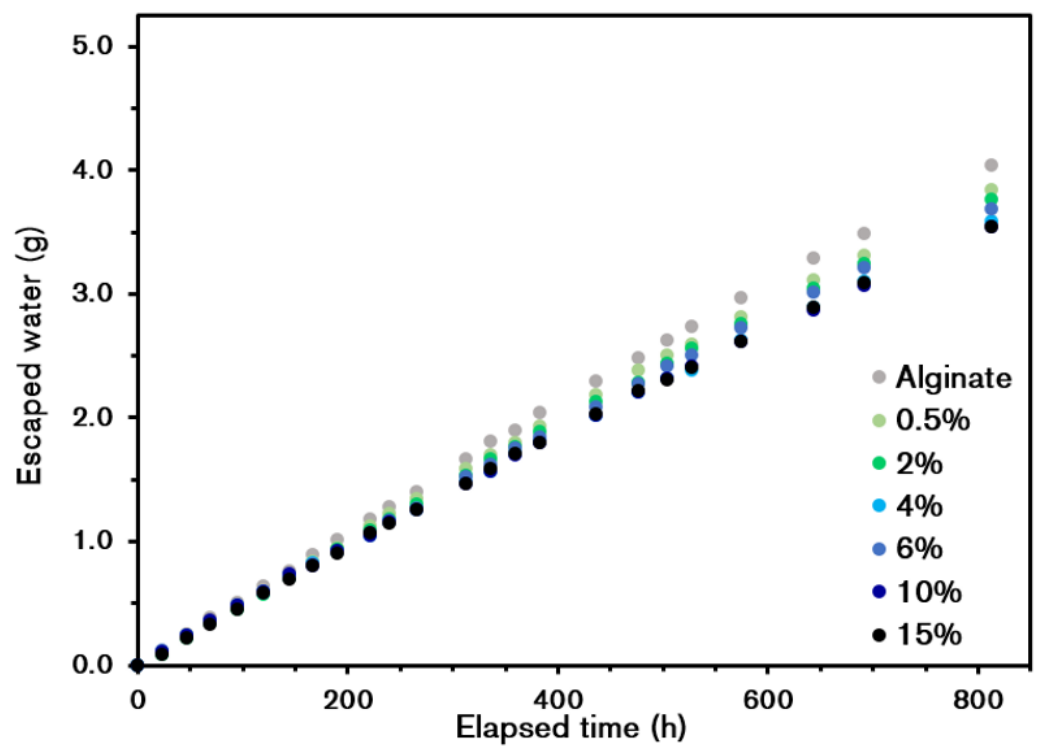

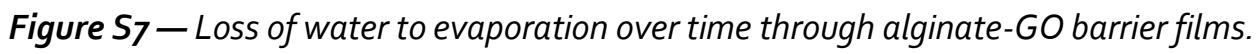

Table S3 - Comparison of barrier performance

\begin{tabular}{cc}
\hline GO weight percent & Permeability $\mathbf{( m g / d \cdot c m} \cdot \mathbf{a t m})$ \\
\hline Alginate & 5.46 \\
$\mathbf{0 . 5 \%}$ & 5.16 \\
$\mathbf{2 \%}$ & 5.09 \\
$\mathbf{4 \%}$ & 4.83 \\
$\mathbf{6 \%}$ & 4.95 \\
$\mathbf{1 0 \%}$ & 4.83 \\
$\mathbf{1 5 \%}$ & 4.79 \\
\hline
\end{tabular}




\section{Contrast enhancement}

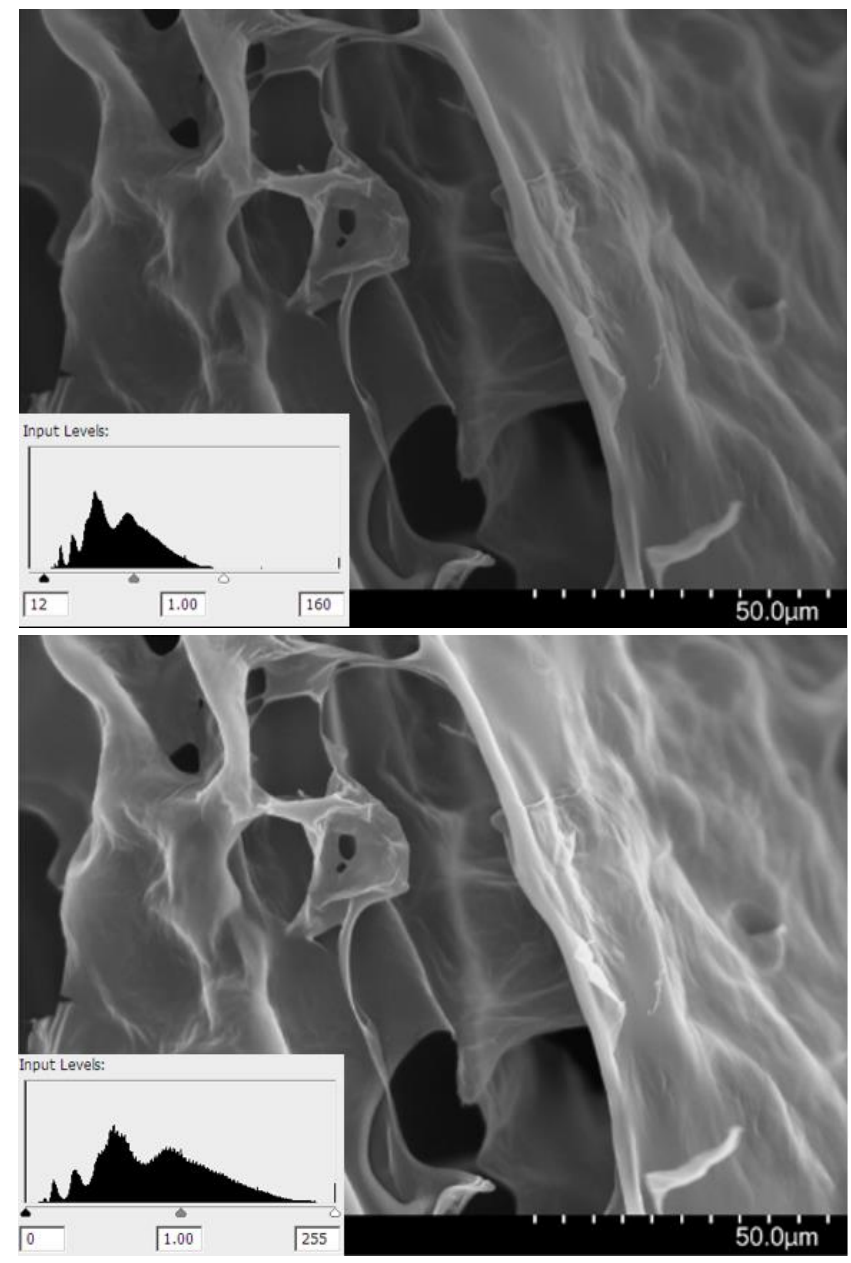

Figure S8 - Demonstration of contrast enhancement, adjusting the output tones with Photoshop to increase tonal range and improve readability.

The contrast in some SEM images was increased to improve readability by expanding the tonal range of the image. This was done using the 'Levels' tool in Adobe Photoshop. First, the histogram was used to identify the tones present in the original image. Then, the output sliders were set so that the edited micrograph's tonal range spanned from black to white (for example, every grey shade darker than $12 / 255$ or lighter than $160 / 255$ in Figure S8 is respectively set to black or white).

The following images have been so enhanced:

Figure 3a (6\%); 3b; 3c

Figure $4 \mathrm{a}(1 \mathrm{~h}, 3 \mathrm{~h}) ; 4 \mathrm{~b}(0.5 \mathrm{~h}, 3 \mathrm{~h})$

The original images are made available in Gallery I. 


\section{Focus stacking}
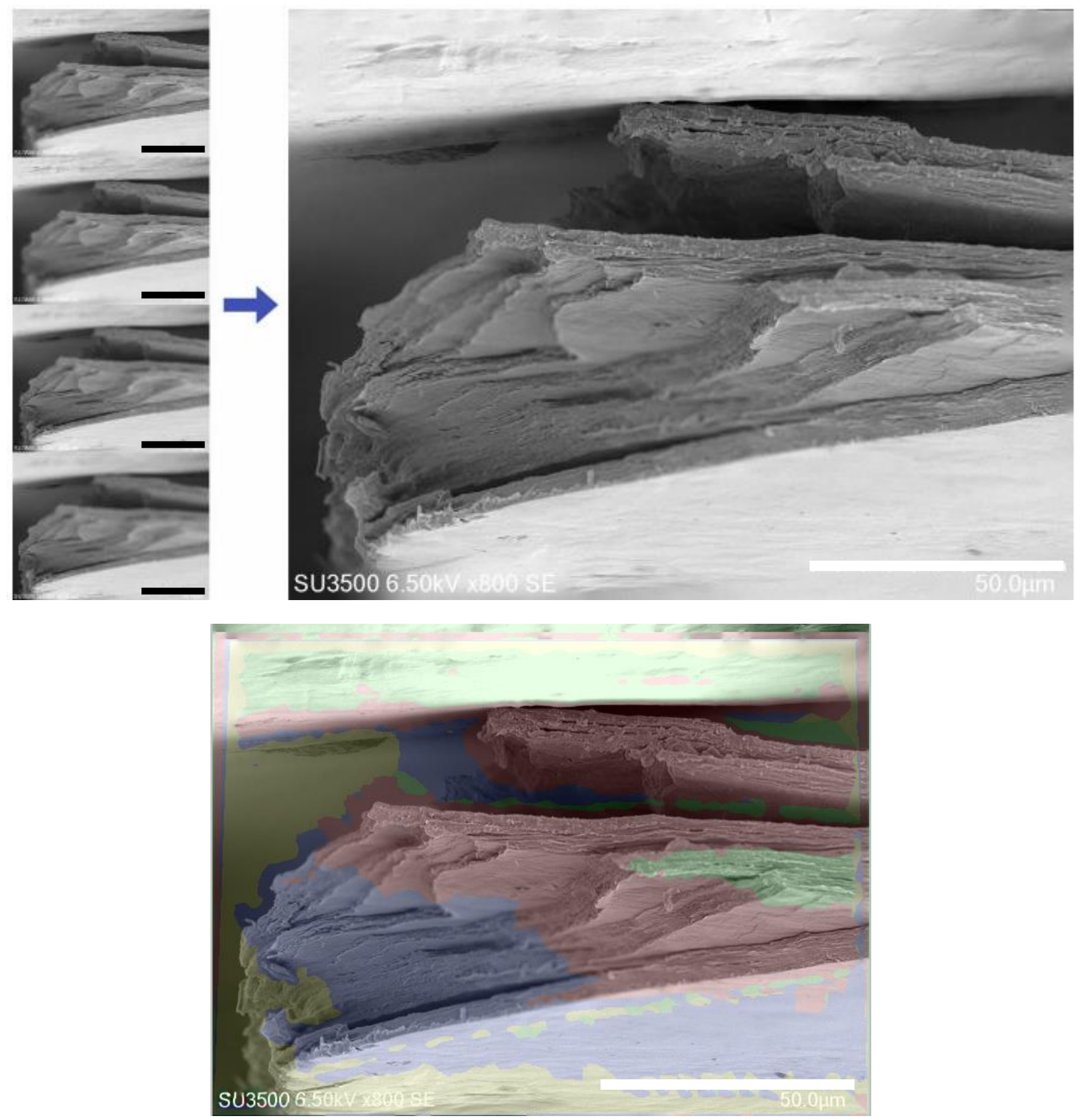

Figure S9 - Demonstration of focus stacking procedure.

The depth of field possible with SEM was insufficient to fully illustrate the fracture interface in

Figure 6. Therefore, multiple exposures of the same scene, with the focus set to different points, were captured and then merged using Photoshop's built-in Auto-Align Layers and Auto-Blend Layers tools. These tools align contents of the separate images, identify the sharpest source image throughout the 
focal range of the scene, and combine these into a single image. Figure S9 illustrates the result, with each colour representing a different source image. The original images are made available in Gallery II. 


\section{Gallery of Original Images}

Gallery I — contrast-enhancement originals

Figure numbers correspond to those in Chapter II.

Figure $3 a(6 \%)$

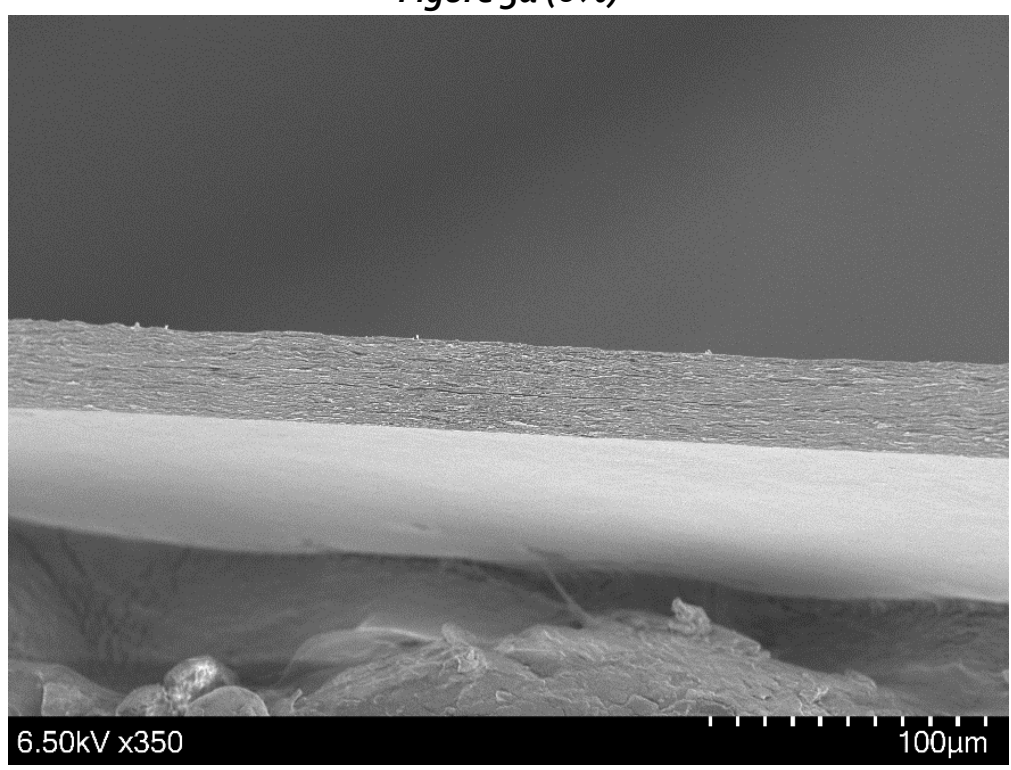

Figure $3 b$

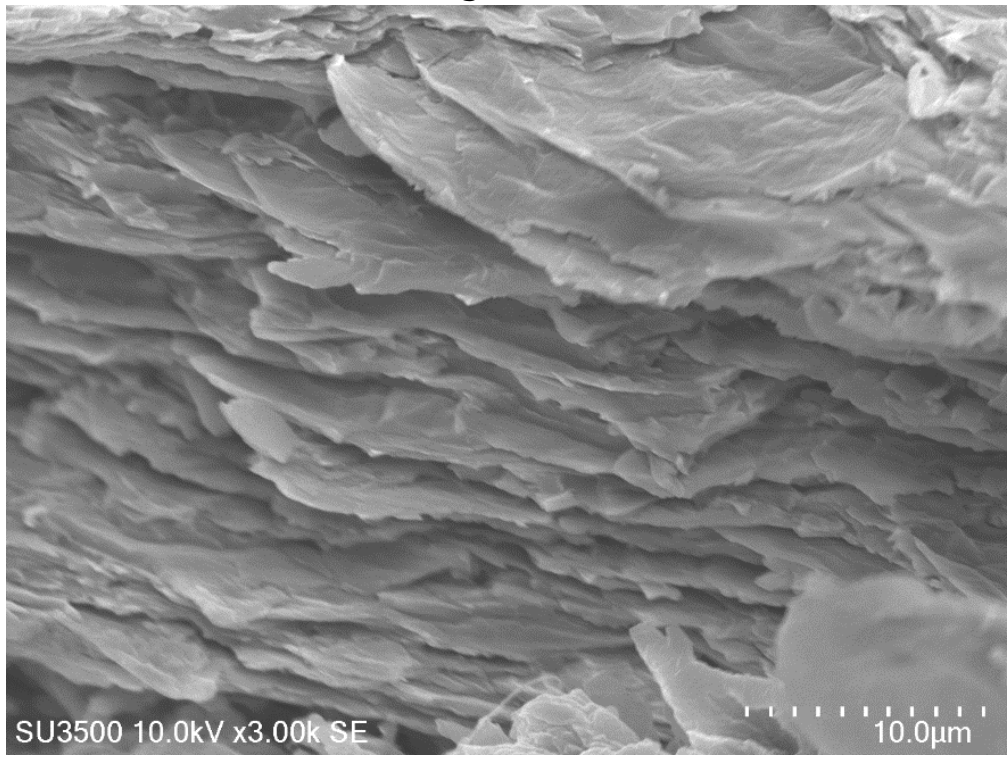


Figure $3 c$

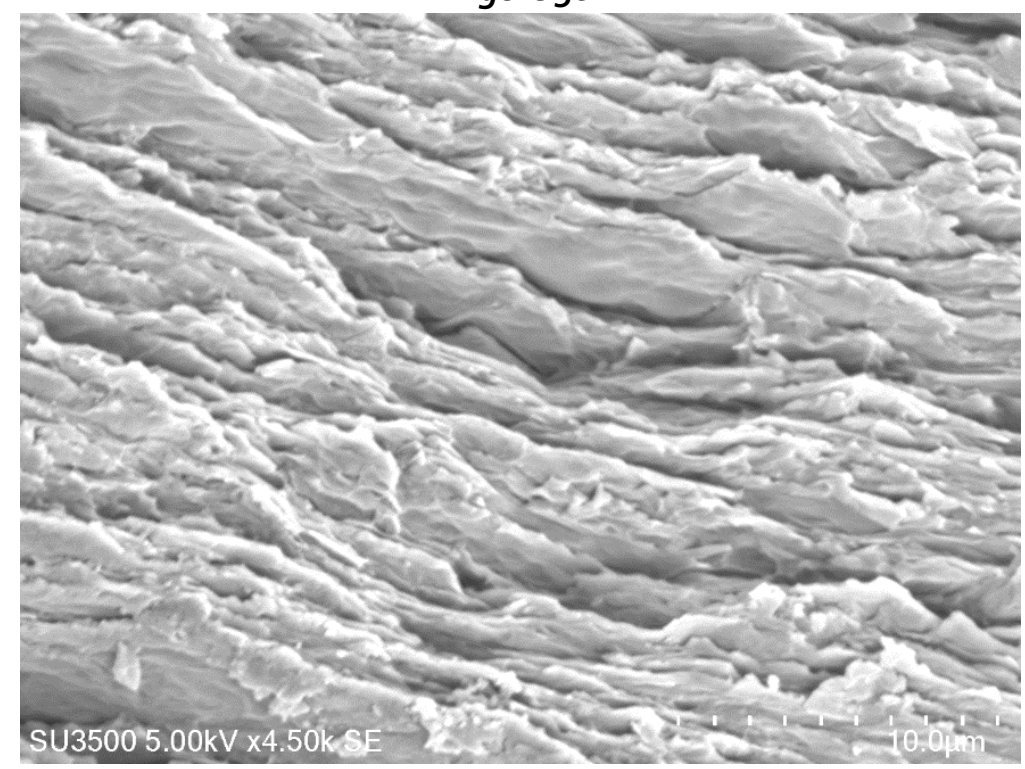

Figure $4 a(1 h)$

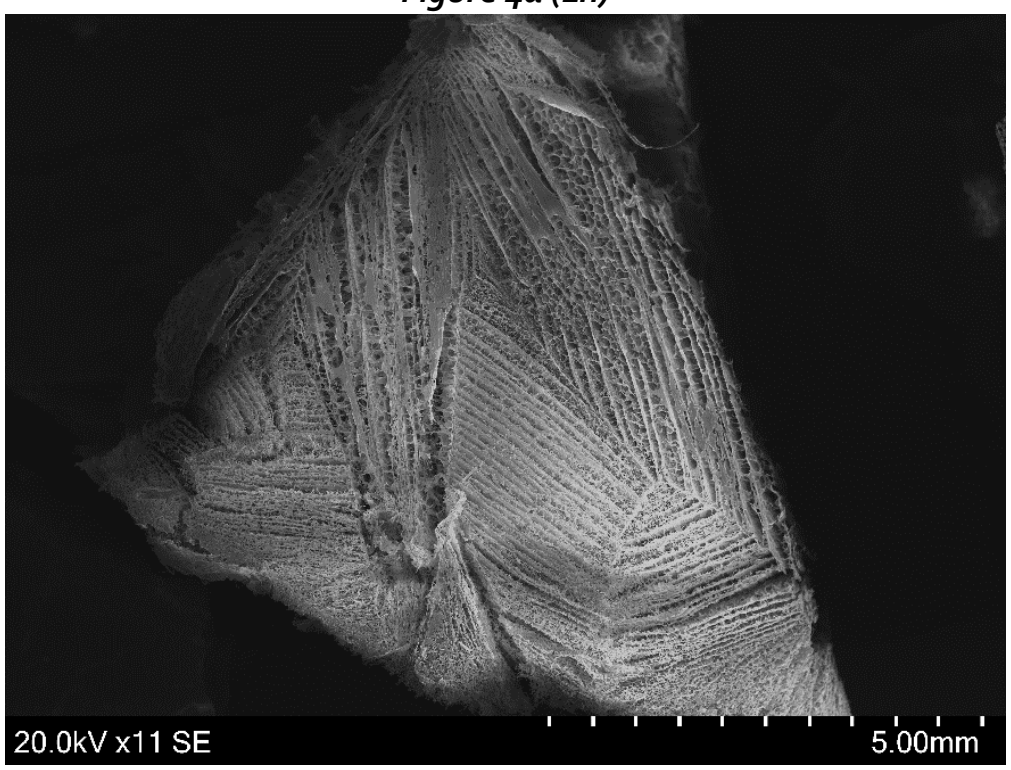


Figure $4 a(3 h)$

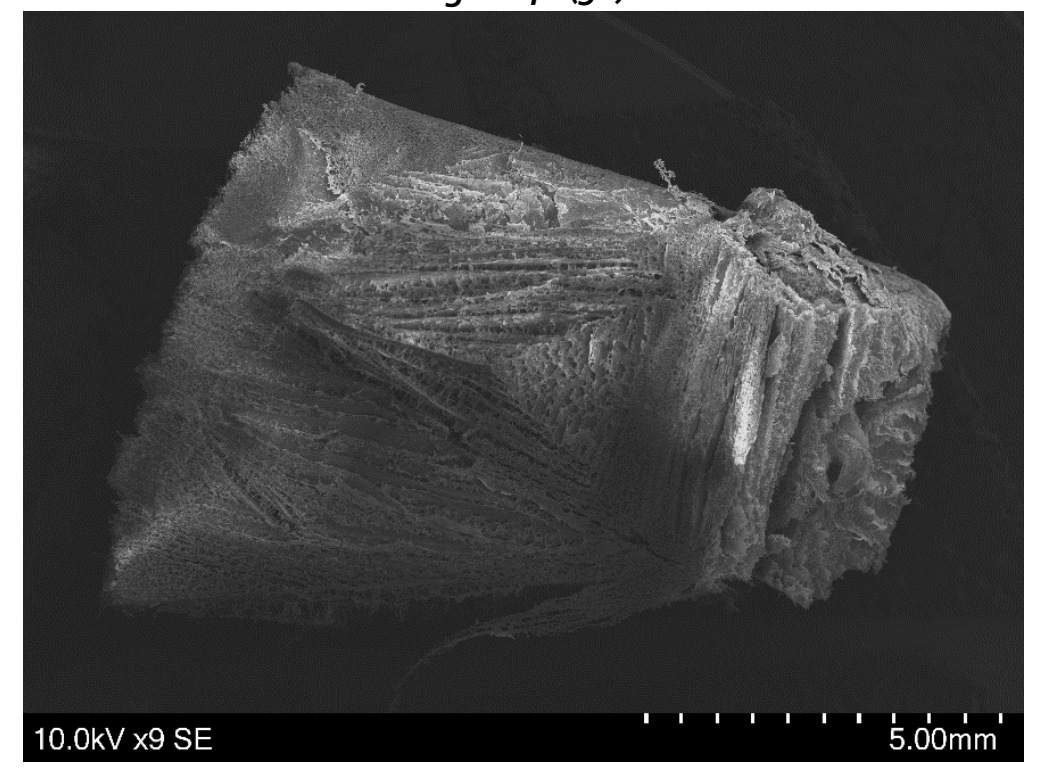

Figure $4 b(0.5 h)$

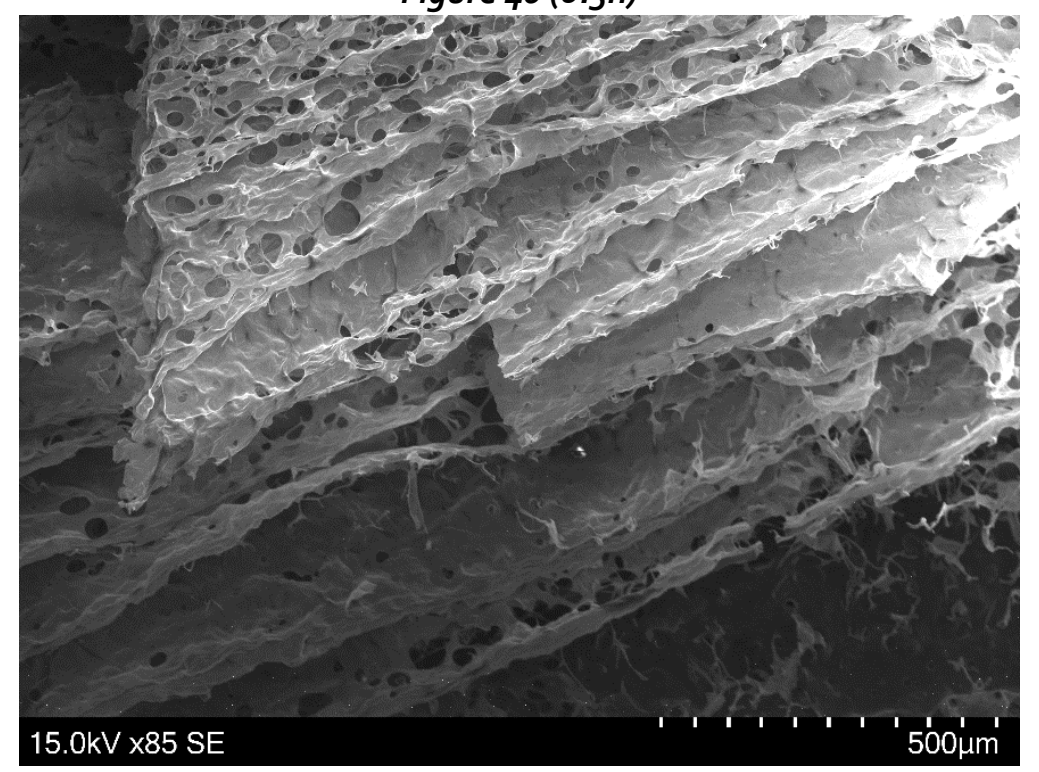


Figure $4 b(3 h)$

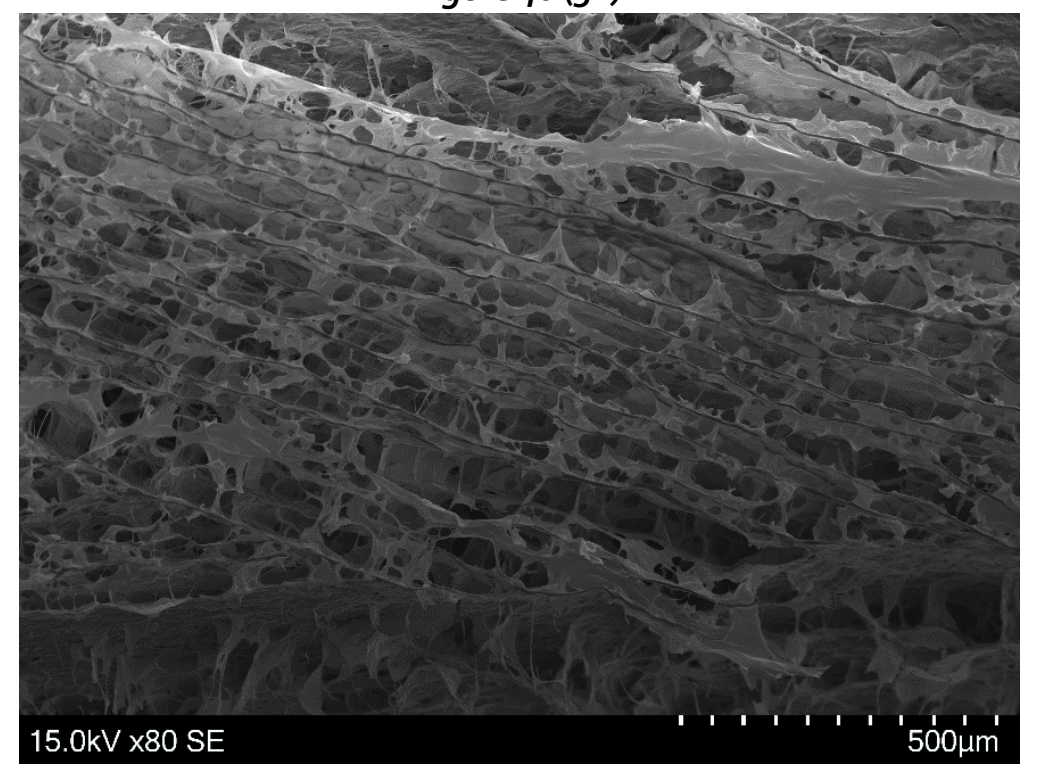


Gallery II — focus stacking originals
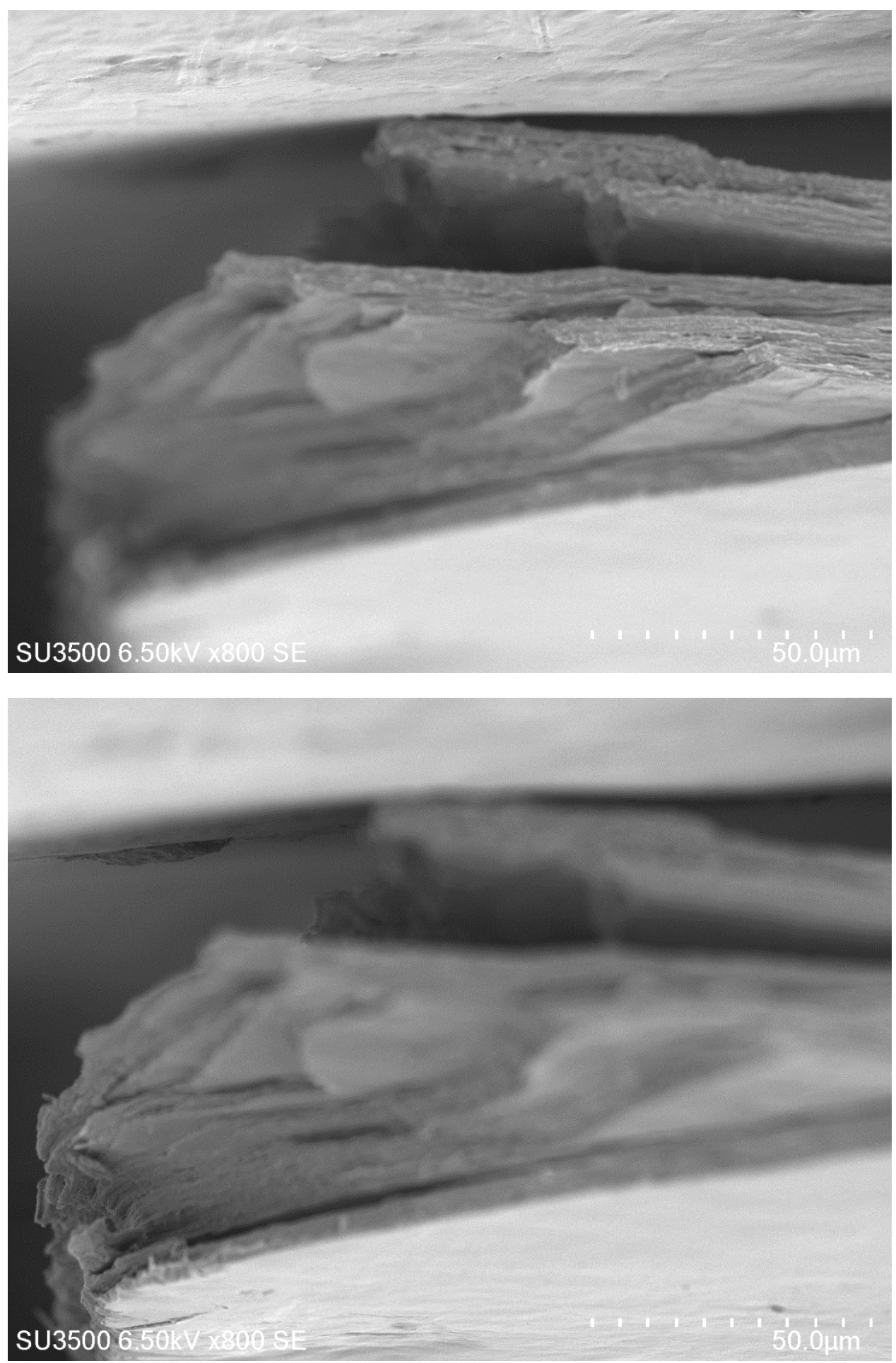

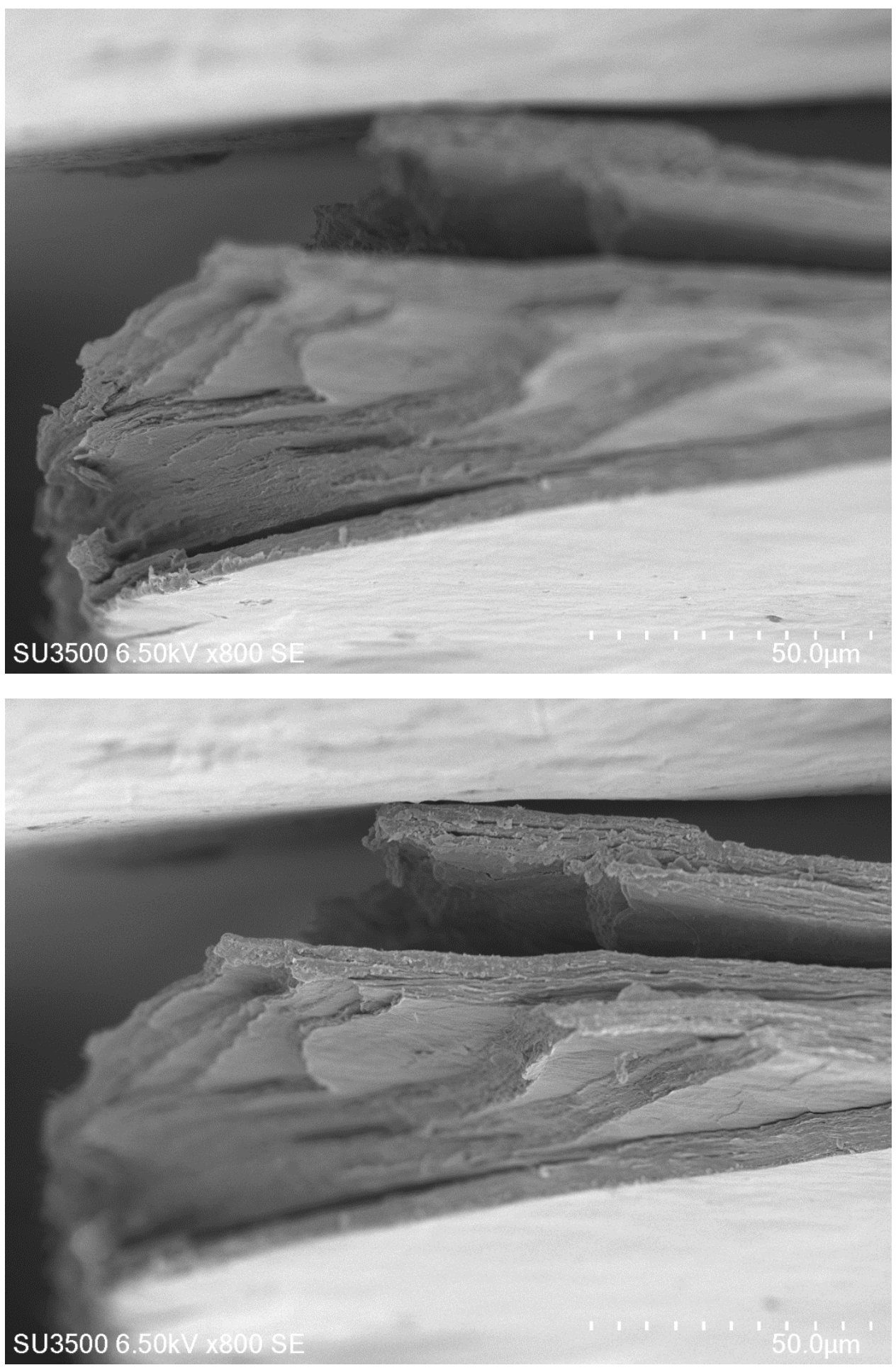


\section{References}

1. Yousefi, N.; Gudarzi, M. M.; Zheng, Q.; Lin, X.; Shen, X.; Jia, J.; Sharif, F.; Kim, J.-K. (2013). Highly aligned, ultralarge-size reduced graphene oxide/polyurethane nanocomposites: Mechanical properties and moisture permeability. Composites Part A 49, 42-50 